\title{
A systematization of the Unscented Kalman Filter theory
}

\author{
Henrique M. T. Menegaz, João Y. Ishihara, \\ Geovany A. Borges, Alessandro N. Vargas
}

\begin{abstract}
In this paper, we propose a systematization of the (discrete-time) Unscented Kalman Filter (UKF) theory. We gather all available UKF variants in the literature, present corrections to theoretical inconsistencies, and provide a tool for the construction of new UKF's in a consistent way. This systematization is done, mainly, by revisiting the concepts of Sigma-Representation, Unscented Transformation (UT), Scaled Unscented Transformation (SUT), UKF, and Square-Root Unscented Kalman Filter (SRUKF). Inconsistencies are related to 1) matching the order of the transformed covariance and cross-covariance matrices of both the UT and the SUT; 2) multiple UKF definitions; 3) issue with some reduced sets of sigma points described in the literature; 4) the conservativeness of the SUT; 5) the scaling effect of the SUT on both its transformed covariance and crosscovariance matrices; and 6) possibly ill-conditioned results in SRUKF's. With the proposed systematization, the symmetric sets of sigma points in the literature are formally justified, and we are able to provide new consistent variations for UKF's, such as the Scaled SRUKF's and the UKF's composed by the minimum number of sigma points. Furthermore, our proposed SRUKF has improved computational properties when compared to state-ofthe-art methods.
\end{abstract}

Index Terms-Unscented Kalman Filter (UKF), Unscented Transformation (UT).

\section{INTRODUCTION}

$\mathbf{S}$ INCE the seminal work of Julier and Uhlmann in 1995 [1], the Unscented Kalman Filter (UKF) has been an object of great interest in the control community. One of the main reasons behind its popularity is the fact that the UKF has provided better results in comparison to the Extended Kalman Filter (EKF) in numerous applications, such as state estimation for battery charging [2], plasma insulins [3], and simultaneous localization and mapping (SLAM) [4], among

Manuscript received October 9, 2013; revised May 23, 2014 and September 26, 2014; accepted January 31, 2015. This work was supported by the Coordination for Improvement of Higher Education Personnel (CAPES) under the Research Grant 00.889.834/0001-08 and Grant 88881.030423/2013-01 and by the National Council for Scientific and Technological Development (CNPQ) under the Grant 312627/2013-0. Recommended by Associate Editor C. M. Lagoa.

H. M. T. Menegaz and G. A. Borges are with Department of Electrical Engineering, University of Brasília, Brasília, DF 70910-900 Brazil (e-mail: henrique.menegaz@lara. unb.br; gaborges@lara.unb.br).

J.Y.Ishihara was with the Basque Center for Applied Mathematics (BCAM), Bilbao, Vizcaya 48009 Spain. He is now with the Department of Electrical Engineering, University of Brasília, Brasília, DF 70910-900 Brazil (e-mail: ishihara@lara.unb.br).

A. N. Vargas was with the Basque Center for Applied Mathematics (BCAM), Bilbao, Vizcaya 48009 Spain. He is now with the Electrotechnical Department at the Federal University of Technology, Cornélio Procópio, PR 86300-000 Brazil (e-mail: avargas@utfpr.edu.br).

Digital Object Identifier: 10.1109/TAC.2015.2404511 others. However, in contrast with the more mathematicallyprincipled approaches utilized in the Gauss-Hermite Filter (GHF) of [5] or the Cubature Kalman Filter (CKF) of [6], all known UKF formulations have had their algorithms originated by ad hoc reasoning following Julier's intuitive principle that "it is easier to approximate a probability distribution than it is to approximate an arbitrary non-linear function" [7]. Although this intuition was suitable for the initial derivation of the filter, its lack of rigor might lead to misleading interpretations and inconsistencies. In fact, some of those have already been reported in the literature. For instance:

- the UKF of [8] presents instability problems (see [9]);

- there are problems with the estimation accuracy of the UKF of [1] (see [10], [11] and Section II-C1);

- the UKF's of [8] and [9] are valid only for scalar systems (see [12] and Sections II-D1 and II-D2).

This work is an attempt to systematize the theory behind the UKF, its first goal being to find a formal justification for the existing UKF definitions. As a result, we unify the most popular discrete-time UKF formulations into a single framework; clarify the reasons behind the aforementioned inconsistencies and propose solutions (see Sections II-B to II-F); and derive new consistent formulations of the UKF (see Section V).

This paper is organized as follows. In Section II, we briefly review non-linear filtering theory and all known discrete-time formulations of the UKF. Next, we discuss previously-known and some new inconsistencies related to these UKF's. From Section III to $\mathrm{V}$, we propose a systematization of the UKF. We introduce the concept of sigma-representations of a random variable (rv) in Section III and present new formalizations for the Unscented Transformation (UT) and the UKF in Sections IV and $\mathrm{V}$, respectively. We conclude with our final remarks in Section VI. Throughout this work, the following notations and definitions are used:

- the set of all real valued rv of length $n$ is denoted by $\Phi^{n}$. For $X \in \Phi^{n}, p_{X}(x)$ is its probability density function (pdf) and $E\{X\}$ or $\bar{X}$, its expected value. If $p_{X}(\bar{X}+$ $x)=p_{X}(\bar{X}-x), \forall x \in \mathbb{R}^{n}$, then $X$ is symmetric. $X \sim$ $\left(m, M_{2}, \ldots, M_{k}\right)^{n}$ stands for a rv $X \in \Phi^{n}$ with mean $m$ and $i$ th central moment $M_{X}^{i}=M_{i}, i=2, \ldots, k$.

- $\otimes$ stands for the Kronecker product operator.

- $\bigotimes_{i=1}^{n} A_{i}:=A_{1} \otimes \cdots \otimes A_{n}$, and $A^{\otimes n}:=\bigotimes_{i=1}^{n} A$.

- $\sqrt{A}$ stands for a square-root matrix of the matrix $A$.

- $[A]_{p \times q}$ stands for a block matrix consisting on the matrix $A$ being repeated $p$ times on the rows and $q$ on the columns. 
- $(A)_{\left(i_{1}: i_{2}\right),\left(j_{1}: j_{2}\right)}$ stands for a sub-matrix of the matrix $A$ formed by the rows $i_{1}$ to $i_{2}$ and the columns $j_{1}$ to $j_{2}$. $(A)_{i, j}$ stands for the $i$ th row and $j$ th column element. $(A)_{* j}$ and $(A)_{i *}$ stand, respectively, for the $j$ th column and $i$ th row of $A$. If no ambiguities exist, we can write $A_{i j}:=(A)_{i, j}$.

- $|A|, A \in \mathbb{R}^{n \times m}$, is such that $|A|_{i j}:=\left|A_{i j}\right|$, where $|\bullet|$ represents the absolute value operator.

- $\operatorname{diag}\left(A_{1}, \ldots, A_{q}\right)$, where $A_{i}$ is square, stands for a block diagonal matrix with each block equal to $A_{i}, i=1, \ldots, q$.

- $\operatorname{sign}(A)$, where $A \in \mathbb{R}^{n \times m}$, is such that $(\operatorname{sign}(A))_{i, j}:=1$, if $A_{i, j} \geq 0$, or $(\operatorname{sign}(A))_{i, j}:=-1$, if $A_{i, j}<0$.

- $\operatorname{tria}\{A\}$ represents a lower triangularization of $A$ (e.g. QR decomposition), and $\operatorname{cdown}\{A, B\}$ the Cholesky factor of $A A^{T}-B B^{T}>0$.

- Tab X [p:q,n:m] refers to the rows $\mathrm{p}$ to $\mathrm{q}$ and the columns $\mathrm{n}$ to $\mathrm{m}$ of Table $\mathrm{X}$. If Table $\mathrm{X}$ has only one column, then $\mathrm{Tab} X[\mathrm{n}: \mathrm{m}]$ refers to its rows $\mathrm{n}$ to $\mathrm{m}$.

- A set $\left\{\chi_{i} \mid \chi_{i} \in \mathbb{R}^{n}\right\}$ generated by a generator $\left[u_{1}, \ldots, u_{\lambda}\right], \lambda \leq n$, is composed by all different permutations of the elements of the vector $\left[u_{1}, \ldots, u_{\lambda},[0]_{1 \times(n-\lambda)}\right]^{T}$. We use the notation $\left\{\chi_{i}\right\}=$ $\operatorname{gen}\left(\left[u_{1}, \ldots, u_{\lambda},[0]_{1 \times(n-\lambda)}\right]\right)$ (see details in [13]).

\section{NONLINEAR AND UnSCENTEd KALMAN FiLTERING}

The Unscented Kalman Filter is a suboptimal solution for the stochastic filtering problem of a discrete-time, dynamical system described either in the additive form

$$
x_{k}=f\left(x_{k-1}, k\right)+q_{k}, \quad y_{k}=h\left(x_{k}, k\right)+r_{k}
$$

or, more generally, in the form

$$
x_{k}=f\left(x_{k-1}, q_{k}, k\right) \quad y_{k}=h\left(x_{k}, r_{k}, k\right)
$$

where $k$ is the time step; $x_{k} \in \mathbb{R}^{n_{x}}$ is the internal state; $y_{k} \in \mathbb{R}^{n_{y}}$ is the measured output; and $q_{k} \in \mathbb{R}^{n_{q}}$ and $r_{k} \in \mathbb{R}^{n_{r}}$ are the process and measurement noises, respectively. The noise terms $q_{k}$ and $r_{k}$ are assumed to be uncorrelated, with means $\hat{q}_{k}=[0]_{1 \times n_{q}}$ and $\hat{r}_{k}=[0]_{1 \times n_{r}}$ and covariance matrices (CM's) $Q_{k}$ and $R_{k}$, respectively.

The stochastic filtering problem consists in finding estimates of the state $x_{k}$ as new measurements $y_{k}$ are acquired. Based on the output history $y_{1: k}:=\left\{y_{i} \mid 1 \leq i \leq k\right\}$, the conditional mean $E\left\{x_{k} \mid y_{1: k}\right\}=\int_{R^{n}} x_{k} p\left(x_{k} \mid y_{1: k}\right) d x_{k}$ is, in general, chosen to be the estimate of $x_{k}$ because $E\left\{x_{k} \mid y_{1: k}\right\}$ is unbiased $\left(E\left\{x_{k}-E\left\{x_{k} \mid y_{1: k}\right\} \mid y_{1: k}\right\}=0\right)$ and it is also an optimal solution with respect to the Minimum Variance (MV) criterion [14], [15]. For linear dynamical systems, the Kalman Filter (KF) provides the optimal solution with respect to the MV criterion, as well as other criteria, when independent Gaussian noise and initial state are considered [14], [16]. However, in the case of non-linear systems, the computation of such optimal solutions tends to be computationally intractable [14], [17], [18]. Therefore, suboptimal approaches must be sought.

Suboptimal, non-linear filters can be classified under four different criteria, at least. A first classification distinguishes the filters approximating the system's functions around the previous estimates (local methods [19])_EKF [18], second order extended Kalman filter (SOEKF) [18], [20]—, from those that do not (global methods) - Gaussian Mixture filter [21], point-mass filter [22], Sequential Monte Carlo Filters (SMCF's) (e.g. Particle Filters, Bootstrap Filters) [23]-[26] and Markov Chain Monte Carlo based filters (MCMCF's) (e.g. filters using Metropolis-Hastings or Gibbs sampling) [27]. A second classification (see [11], [19]) is based on whether there is the necessity of calculating derivatives of system functions-EKF and SOEKF-or not, i.e., if the filter is derivative-free-UKF [1], [7], GHF [5], Central Difference Filter (CDF) [5], Divided Difference filter (DDF) [28], and CKF [6], [29]. A third classification considers filters for which statistics of posterior random variables (rv's) are obtained by sampling of previous pdf's. The sampling can be stochastic, as in SMCF's and MCMCF's, or deterministic, as in the UKF and the DDF. Monte Carlo (MC) methods consist, essentially, on taking a very large quantity of samples of the rv of interest in a random fashion [23]-[27], while sigma point methods consist on analytically choosing a finite number of weighted samples [30]. A fourth classification takes into account if the posterior estimate of a filter is based on a Gaussian assumption (EKF, SOEKF, UKF's, GHF, CDF, DDF, CKF) or not (Gaussian Mixture filter, point-mass filter, SMCF's, MCMCF's).

Among all non-linear filters, the EKF is the most widelyknown and implemented in practical applications [7], [14], [18]. It is obtained as the first order truncation of the Taylor series approximation of system's non-linear dynamics, retaining the same prediction-correction structure as the KF for linear dynamical systems. Although several filters in the literature have been proposed in order to improve upon computational aspects related to the EKF, it was just recently that UKF's have become noticeable as a competitive and preferable alternative [7], [30]. Among the positive features related to the UKF, one can mention:

- the computational complexities of the UKF's and of the EKF are of the same order- $\mathcal{O}\left(n^{3}\right)-$, but UKF's tend to attain better estimation performance;

- the UKF is derivative-free (no need to compute Jacobians), while the EKF requires the dynamics to be differentiable. Thus, unlike the EKF, UKF's can be used in situations where Jacobians may not always exist, such as systems with discontinuities (cf. [7]).

These and other good properties related to the UKF have become well-known since its introduction (see more details in Section II-B). However, later UKF variants have been reported to be inconsistent (see Sections II-C to II-F) and it is currently difficult to assess whether these inconsistencies are present in all UKF variants. Seeking to provide clarifications, we first review all main UKF variants in the next subsection.

\section{A. Unscented Kalman Filters}

All UKF variants, as in the EKF, keep the structure of the Kalman filter for linear systems of one prediction (or $a$ priori estimation) and one correction (or update) step. This can be seen, for instance, in the UKF of [31]: consider (1) and suppose that, at time step $k, \hat{x}_{k-1 \mid k-1}$ and $\hat{P}_{x x}^{k-1 \mid k-1}$ are 
given. Choose a real $\kappa>-n_{x}$ and define, for $1 \leq i \leq n_{x}$, the weights and points

$$
\begin{aligned}
& w_{0}:=\frac{\kappa}{n_{x}+\kappa}, w_{i}=w_{i+n_{x}}:=\frac{1}{2\left(n_{x}+\kappa\right)}, \chi_{0}^{k-1 \mid k-1}:=\hat{x}_{k-1 \mid k-1} \\
& \chi_{i}^{k-1 \mid k-1}:=\hat{x}_{k-1 \mid k-1}+\left(\sqrt{\left(n_{x}+\kappa\right) \hat{P}_{x x}^{k-1 \mid k-1}}\right)_{* i} \\
& \chi_{i+n_{x}}^{k-1 \mid k-1}:=\hat{x}_{k-1 \mid k-1}-\left(\sqrt{\left(n_{x}+\kappa\right) \hat{P}_{x x}^{k-1 \mid k-1}}\right)_{* i}
\end{aligned}
$$

For $0 \leq i \leq 2 n_{x}$, define the transformed sigma points

$$
\chi_{i}^{k \mid k-1}:=f\left(\chi_{i}^{k-1 \mid k-1}, k\right), \gamma_{i}^{k \mid k-1}:=h\left(\chi_{i}^{k \mid k-1}, k\right)
$$

and their associated statistics $\left((A)(\diamond)^{T}\right.$ stands for $\left.(A)(A)^{T}\right)$

$$
\begin{aligned}
& \hat{x}_{k \mid k-1}:=\sum_{i=0}^{2 n_{x}} w_{i} \chi_{i}^{k \mid k-1}, \quad \hat{y}_{k \mid k-1}:=\sum_{i=0}^{2 n_{x}} w_{i} \gamma_{i}^{k \mid k-1} \\
& \hat{P}_{x x}^{k \mid k-1}:=\sum_{i=0}^{2 n_{x}} w_{i}\left(\chi_{i}^{k \mid k-1}-\hat{x}_{k \mid k-1}\right)(\diamond)^{T}+Q_{k} \\
& \hat{P}_{x y}^{k \mid k-1}:=\sum_{i=0}^{2 n_{x}} w_{i}\left(\chi_{i}^{k \mid k-1}-\hat{x}_{k \mid k-1}\right)\left(\gamma_{i}^{k \mid k-1}-\hat{y}_{k \mid k-1}\right)^{T}
\end{aligned}
$$

along with the innovation's covariance

$$
\hat{P}_{y y}^{k \mid k-1}:=\sum_{i=0}^{2 n_{x}} w_{i}\left(\gamma_{i}^{k \mid k-1}-\hat{y}_{k \mid k-1}\right)(\diamond)^{T}+R_{k} .
$$

Finally, instantiate the KF's correction equations

$$
\begin{gathered}
G_{k}:=\hat{P}_{x y}^{k \mid k-1}\left(\hat{P}_{y y}^{k \mid k-1}\right)^{-1}, \hat{x}_{k \mid k}:=\hat{x}_{k \mid k-1}+G_{k}\left(y_{k}-\hat{y}_{k \mid k-1}\right) \\
\hat{P}_{x x}^{k \mid k}:=\hat{P}_{x x}^{k \mid k-1}-G_{k} \hat{P}_{y y}^{k \mid k-1} G_{k}^{T} .
\end{gathered}
$$

All popular variants of the UKF for discrete-time systems have the same prediction-correction structure of the UKF of [31], but with different expressions for (3)-(7). They can be classified according to a few different criteria.

A first criterion is related to the form of the underlying dynamical system, i.e., whether the system is described in additive form (1) or in the more general form (2). Depending on the case, the a priori $\mathrm{rv}$ is either the previous state estimate $\hat{x}_{k-1 \mid k-1}$ with estimate CM $\hat{P}_{x x}^{k-1 \mid k-1}$ (as in the UKF of [31] above) or the previous augmented vector estimate $\hat{x}_{k-1 \mid k-1}^{a}$ with dimension $n_{a}=n_{x}+n_{q}+n_{r}$, which is defined as $\hat{x}_{k-1 \mid k-1}^{a}:=\left[\hat{x}_{k-1 \mid k-1}^{T}, \hat{q}_{k}^{T}, \hat{r}_{k}^{T}\right]^{T}$ with covariance matrix and square-root covariance matrix

$$
\begin{aligned}
& \hat{P}_{x x}^{a, k-1 \mid k-1}:=\operatorname{diag}\left(\hat{P}_{x x}^{k-1 \mid k-1}, Q_{k}, R_{k}\right) \\
& \sqrt{\hat{P}_{x x}^{a, k-1 \mid k-1}}:=\operatorname{diag}\left(\sqrt{\hat{P}_{x x}^{k-1 \mid k-1}}, \sqrt{Q_{k}}, \sqrt{R_{k}}\right) .
\end{aligned}
$$

Although it is always possible to use the general form of the UKF for either (1) or (2), the additive form of the UKF is preferable for (1) because its algorithm is computationally less expensive than the corresponding UKF in general form. Nevertheless, as pointed out by [32], the additive version yields the same result as the augmented one only if the predicted sigma points $\chi_{i}^{k \mid k-1}$ are resampled from the previous mean $\hat{x}_{k \mid k-1}$ and covariance matrix $\hat{P}_{x x}^{k \mid k-1}$ when performing the UT in the correction step. If correlated noises are considered, the general form for the UKF with a simple change in (8)-(9) (see [7]) should be used for either (1) or (2).
Remark 1 Filters for system descriptions besides (1) and (2) can be easily obtained. For partially-additive dynamical systems, where either the process or the measurement equations are given in additive form and the other is given in the general form, the augmented state vector of the filter is composed only by the noise of whatever function is in general form [15]. For partially nonlinear dynamical systems, where one of these equations is linear, the linear $K F$ equations can usually be used in the part of the UKF referring to the linear equation [33].

A second major criterion to classify UKF variants is the propagation form of CM's in the algorithm for the filter. The UKF can be in the covariance form, where the CM's are calculated directly, or in the square-root form, where the square-root of the CM's are computed recursively instead of propagating the CM's themselves.

Let us first consider the UKF algorithms in the covariance form. In these algorithms, the mean and the CM of the predicted and corrected variables are approximated using a so-called Unscented Transformation of the previous estimate. Any UT variant is based on some set of sigma points which is chosen using a given mean and CM information. Roughly, an UT approximates the joint pdf of two rv's $X \sim\left(\bar{X}, P_{X X}\right)^{n}$ and $Y \sim\left(\bar{Y}, P_{Y Y}\right)^{n_{y}}$ related by a given function $Y=F(X)$ by introducing the sets of weighted points (the sigma points) $\sigma_{\chi}=\left\{\chi_{i}, w_{i}^{m}, w_{i}^{c}\right\}_{i=1}^{N}$ and

$$
\sigma_{\gamma}=\left\{\gamma_{i}, w_{i}^{m}, w_{i}^{c} \mid \gamma_{i}=F\left(\chi_{i}\right)\right\}_{i=1}^{N}
$$

with the following sample statistics:

$$
\begin{aligned}
& \mu_{\chi}:=\sum_{i=1}^{N} w_{i}^{m} \chi_{i}, \Sigma_{\chi \chi}:=\sum_{i=1}^{N} w_{i}^{c}\left(\chi_{i}-\mu_{\chi}\right)(\diamond)^{T} \\
& \mu_{\gamma}:=\sum_{i=1}^{N} w_{i}^{m} \gamma_{i}, \Sigma_{\gamma \gamma}:=\sum_{i=1}^{N} w_{i}^{c}\left(\gamma_{i}-\mu_{\gamma}\right)(\diamond)^{T} \\
& \Sigma_{\chi \gamma}:=\sum_{i=1}^{N} w_{i}^{c}\left(\chi_{i}-\mu_{\chi}\right)\left(\gamma_{i}-\mu_{\gamma}\right)^{T} .
\end{aligned}
$$

For any UT variant, the points and weights in the sigma set $\sigma_{\chi}$ are required $^{1}$ to be such that $\mu_{\chi}=\bar{X}, \Sigma_{\chi \chi}=P_{X X}$ and, as consequence, $\mu_{\gamma}$ and $\Sigma_{\gamma \gamma}$ are expected to be, respectively, equal to $\bar{Y}$ and $P_{Y Y}$ up to their second order Taylor approximations [7]. In additive UKF's for (1), generally two UT's are performed: one for $F(X)=f(X, k)+q_{k}$ with $X$ being the previous estimate $\hat{x}_{k-1 \mid k-1}$, and another for $H(X)=h(X, k)+r_{k}$ with $X$ being $\hat{x}_{k \mid k-1}$. For general UKF's, one has UT for $F(X)$ with $X=\hat{x}_{k \mid k-1}^{a}$ and for $H(X)$ with $X=\hat{x}_{k \mid k-1}^{a}$.

Variants of the UKF can be classified according to the underlying UT used. The UT variants differ from each other depending on how the transformed statistics are calculated and by their choice of the sigma set $\sigma_{\chi}$. All basic sigma set variants in the literature are presented in Table I. The ones from [1] and [7] are equivalent if one chooses $w_{0}=\kappa /(\kappa+n)$ (cf. Tab I [1:2]). Hence, we can say that the general and additive UKF's of [1] and [7] are equivalent (cf. Tab III [1:2,1:3] and Remark 3). The basic difference is in their choice of the tuning parameter $w_{0}$ or $\kappa$.

\footnotetext{
${ }^{1}$ In the literature, these requirements are said to be properties of UT's. In Section II-C, we present several counter-examples to these claims.
} 
TABLE I

SETS OF SIGMA POINTS FOR $X \sim\left(\bar{X}, P_{X X}\right)^{n}$

\begin{tabular}{l} 
Symmetric set of $\underline{[1]}(N=2 n+1)$. Choose $\kappa>-n$. Set \\
$w_{0}=\frac{\kappa}{n+\kappa}, \chi_{0}=\bar{X}$ and, for $i=1, \ldots, n: w_{i}=w_{i+n}=\frac{1}{2(n+\kappa)}$ \\
$\Delta \chi_{i}=\left(\sqrt{(n+\kappa) P_{X X}}\right)_{* i}, \chi_{i}=\bar{X}+\Delta \chi_{i}, \chi_{i+n}=\bar{X}-\Delta \chi_{i}$. \\
\hline Symmetric set of $\underline{[7]}(N=2 n+1)$. Choose $w_{0}<1$. Set \\
$\chi_{0}=\bar{X}$, and, for $i=1, \ldots, n,: w_{i}=w_{i+n}=\frac{1-w_{0}}{2 n}$, \\
$\Delta \chi_{i}=\left(\sqrt{\frac{n}{1-w_{0}} P_{X X}}\right)_{* i}, \chi_{i}=\bar{X}+\Delta \chi_{i}, \chi_{i+n}=\bar{X}-\Delta \chi_{i}$,
\end{tabular}

Reduced set of [8] $(N=n+1)$. Choose $0 \leq w_{0}<1$. Define

$w_{2}=w_{1}=\frac{1-w_{0}}{2^{n}} ; w_{i}=2^{i-1} w_{1}$, for $i=3, \ldots, n+1 ; \chi_{0}^{1}=[0]$

$\chi_{1}^{1}=\left[-1 / \sqrt{2 w_{1}}\right] ; \quad \chi_{2}^{1}=-\chi_{1}^{1}$; for $j=1, \ldots, n$ :

$\chi_{0}^{j+1}=\left[\chi_{0}^{j}, 0\right]^{T}, \chi_{i}^{j+1}=\left[\chi_{i}^{j}, \frac{-1}{\sqrt{2 w_{j}}}\right]^{T}$,

$\chi_{j+1}^{j+1}=\left[[0]_{1 \times j}, \frac{1}{\sqrt{2 w_{j}}}\right]^{T}, \chi_{i}:=\sqrt{P_{X X}} \chi_{i}^{n+1}+\bar{X}$.

Spherical simplex set of [9] $(N=n+2)$. Choose $0 \leq w_{0} \leq 1$. Set: $w_{i}=\frac{1-w_{0}}{n}, i=1, \ldots, n+1 ; \chi_{0}^{1}=[0], \chi_{1}^{1}=\left[\frac{1}{\sqrt{2 w_{1}}}\right], \chi_{2}^{1}=-$ $\chi_{1}^{1}$; for $j=1, \ldots, n: \chi_{0}^{j+1}=\left[\chi_{0}^{j-1}, 0\right]^{T}, a_{i}=\frac{1}{\sqrt{\left(j^{2}+j\right) w_{1}}}, \chi_{i}^{j+1}=$ $\left[\chi_{i}^{j-1},-a_{i}\right]^{T}, \chi_{j+1}^{j+1}=\left[[0]_{1 \times(j-1)}, a_{i}\right]^{T} \chi_{i}:=\sqrt{P_{X X}} \chi_{i}^{n+1}+\bar{X}$ Simplex set of [34] $(N=n+1)$. Set: $w_{i}=\frac{1}{n+1}, i=1, \ldots, n+1$; $\Gamma=\left[\Gamma_{1 *}^{T}, \ldots, \Gamma_{n *}^{T}\right]^{T}$, for $\Gamma_{j *}=\sqrt{n+1}\left[\left[\sqrt{\frac{j}{j+1}}\right]_{1 \times j},-\sqrt{\frac{j+1}{j}}\right.$, $\left.[0]_{1 \times(n-j)}\right],\left[\chi_{1}, \ldots, \chi_{n+1}\right]:=\sqrt{P_{X X}} \Gamma+[\bar{X}]_{1 \times(n+1)}$.

Minimum set of [12] $(N=n+1)$. Choose $0<w_{(n+1)}<1$. Set $\rho:=\sqrt{\frac{1-w_{(n+1)}}{n}}, C:=\sqrt{I_{n}-\rho^{2} \underline{[1]}_{n \times n}}, W=\operatorname{diag}\left(w_{1}, \ldots\right.$, $\left.w_{n}\right)$. For $i=1, \ldots, n: w_{i}=\left(C^{-1} w_{(n+1)} \rho^{2} \underline{[1]}_{n \times n}\left(C^{T}\right)^{-1}\right)_{i, i}$, $\chi_{i}:=\left(\sqrt{P_{X X}} C(\sqrt{W})^{-1},-\rho \sqrt{P_{X X}} \frac{[1]_{n \times 1}}{\sqrt{w_{(n+1)}}}\right)_{* i}+\bar{X}$.

Symmetric set of [35] $(N=2 n+1)$. Choose $\alpha, \kappa \in \mathbb{R}$. Set $\lambda=$ $\alpha^{2}(n+\kappa)-n>-n, \chi_{0}=\bar{X}, w_{0}^{c}=\frac{\lambda}{n+\lambda}+\left(1-\alpha^{2}+\beta\right)$; $w_{0}^{m}=\frac{\lambda}{n+\lambda}$ for $1 \leq i \leq n: w_{i}^{m}=w_{i+n}^{m}=w_{i}^{c}=w_{i+n}^{c}=\frac{\lambda}{n+\lambda}$, $\Delta \chi_{i}=\left(\sqrt{(n+\lambda) P_{X X}}\right)_{* i}, \chi_{i}=\bar{X}+\Delta \chi_{i}, \chi_{i+n}=\bar{X}-\Delta \chi_{i}$.

Fifth order set of [36] $\left(N=2 n^{2}+1\right)$. Set: $w_{i}=\frac{1}{36}$, for $2 n+1$ $\leq i \leq 2 n^{2} ; w_{i}=\frac{4-n}{18}$, for $1 \leq i, \leq 2 n ; w_{2 n^{2}+1}=\frac{n^{2}-7 n}{18}+1$; $\left\{\xi_{i}\right\}_{i=1}^{2 n}=\operatorname{gen}([ \pm \sqrt{3}]),\left\{\xi_{i}\right\}_{i=2 n+1}^{2 n^{2}}=\operatorname{gen}([ \pm \sqrt{3}, \pm \sqrt{3}])$, $\xi_{2 n^{2}+1}=[0]_{n \times 1}$; for $i=1, \ldots, 2 n^{2}+1$, set $\chi_{i}=\bar{X}+\sqrt{P_{X X}} \xi_{i}$. Set of [37] $\left(N=\varphi^{n}\right)$. Choose $\varphi \in \mathbb{N}$. For $j=1, \ldots, \varphi ; i=1, \ldots, n$, set: $F(x)=\frac{1}{\sqrt{2 \pi}} \int_{\infty}^{x} e^{-\frac{u^{2}}{2}} d u ; b_{i}=F^{-1}\left(\frac{2 i-1}{2 \varphi}\right) ; c_{j_{i}}=$ $\sqrt{\frac{\varphi}{\sum_{j=1}^{\varphi} b_{j}}} b_{i} ; w_{j_{i}}=\frac{1}{\varphi^{n}} ; \chi_{j_{i}}=\bar{X}+\sum_{i=1}^{n} c_{j_{i}} \sqrt{\lambda_{i}} v_{i}$, where $\lambda_{i}$ is an eigenvalue and $v_{i}$ an eigenvector of $P_{X X}$.

One can also consider the geometrical distribution of the sigma points. For points $\chi_{i}$ symmetrically distributed in a geometrical sense, we have the UKF's [1], [7], [35], [36]. For asymmetric sets, one can mention the reduced of [8]; the spherical simplex of [9]; the simplex of [34]; and the minimum of [12].

Another classification criterion considers the number of sigma points in the sigma set $\sigma_{\chi}$ for the previous variable $X \in \Phi^{n}$. As can be seen by Table I, among the major general and additive UKF variants that use symmetric sets, the UKF's of [1], [7], and [35] use $\sigma_{\chi}$ with $N=2 n+1$ points. Among the major general and additive UKF variants that use asymmetric sets, the UKF's of [9] use $\sigma_{\chi}$ with $N=n+2$ points and the UKF's of [8], [34], and [12] use $\sigma_{\chi}$ with $N=n+1$ sigma points, which is the minimum amount [8]. Finally, the UKF of [36] uses $\sigma_{\chi}$ with $N=2 n^{2}+1$ points and the one of [37] uses $N=\varphi^{n}, \varphi \in \mathbb{N}$.

One can consider which moments of the sigma set $\sigma_{\chi}$ are
TABLE II

OTHER UNSCENTED TRANSFORMATIONS

\begin{tabular}{cc}
\hline & Scaled UT $[\mathbf{3 8}]$ \\
& Obtain previous $\left\{\chi_{i}, w_{i}\right\}_{i=1}^{N}$. Choose $\alpha \geq 0$. Set, for $2 \leq i \leq N:$ \\
& $w_{1}^{\prime}=w_{1} \alpha^{-2}+1-\alpha^{-2} ; \chi_{i}^{\prime}=\chi_{1}+\alpha\left(\chi_{i}-\chi_{1}\right) ; w_{i}^{\prime}=w_{i} \alpha^{-2}$. \\
\hline 2 & Transformed set: $\left\{\gamma_{i}, w_{i}^{\prime} \mid \gamma_{i}=F\left(\chi_{i}^{\prime}\right)\right\}_{i=1}^{N} \cdot$ \\
3 & Transformed CM: $\Sigma_{\gamma \gamma}^{*}:=\sum_{i=0}^{N} w_{i}^{\prime}\left(\gamma_{i}-\mu_{\gamma}\right)(\diamond)^{T}+$ \\
& $\left(1+\beta-\alpha^{2}\right)\left(\gamma_{0}-\mu_{\gamma}\right)(\diamond)^{T}$. \\
\hline & Auxiliary form of the UT (AuxUT) $[\mathbf{3 8}]$ \\
4 & $g(X, c, \alpha, \kappa):=\kappa^{-1} F(c+\alpha(X-c))-\kappa^{-1} F(c)+F(c)$. \\
\hline 5 & Obtain previous $\left\{\chi_{i}, w_{i}\right\}_{i=1}^{N}$. Choose $\alpha \geq 0$. Define \\
& Transformed set: $\left\{\gamma_{i}, w_{i} \mid \gamma_{i}=g\left(\bar{X}, \mu_{\chi}, \alpha, \alpha^{2}\right)\right\}_{i=1}^{N} \cdot$ \\
6 & Transformed CM: $\Sigma_{\gamma \gamma}^{*}:=\alpha^{2} \sum_{i=0}^{N} w_{i}\left(\gamma_{i}-\mu_{\gamma}\right)(\diamond)^{T}$. \\
\hline
\end{tabular}

equal to the moments of the previous $\operatorname{rv} X$. The sets $\sigma_{\chi}$ of the UKF's of [1], [7], [8], [12], [34], [35] and [36] match the first two moments of any $X$. If $X$ is symmetric, then the sets $\sigma_{\chi}$ in the UKF's of [1], [7], [35] and [36] match also all odd moments. If $X$ is Gaussian, then $\sigma_{\chi}$ in the UKF of [36] further matches the fourth moment of $X$ and the one of [37] matches the first two moments of $X$. However, not all UKF variants have their $\sigma_{\chi}$ matching the first and second moments of $X$ (see Section II-D).

Considering how the transformed statistics are calculated, one can distinguish two more UT's other than the first six that arrive from the different choices of $\sigma_{\chi}$ (the ones of Table I): the AuxUT of [38] and the scaled UT of [38] (cf. Table II). Their sigma sets are scaling transformations of a previous sigma set and the statistics calculations are modified versions of (11)(13) (cf. Table II). According to [38], the auxiliary and the scaled forms yield the same results. Note that [39] presented an embryonic form of these scaling unscented forms.

In summary, all additive UKF's in covariance form in the literature are represented in Table III using the sigma sets of Table I and the UT's of Table II and (10)-(13) [all UKF variants for the general case (2) can be obtained similarly with a corresponding slightly modified version of Table III].

Remark 2 Some particularities of Tables I, II, and III should be highlighted. If, for example, one wished to implement the UKF of [31], it would be necessary to choose $w_{i}^{m}=w_{i}$ when calculating any mean and $w_{i}^{c}=w_{i}$ when calculating any covariance matrix. As an example of how these tables can be used, the additive form UKF of [31], (3)-(6), can be obtained by taking Tab III [1,1:3]. The previous set of this filter is the symmetric set of [1] calculated for $\bar{X}=\hat{x}_{k-1 \mid k-1}$ and $P_{X X}=\hat{P}_{x x}^{k-1 \mid k-1}$.

Remark 3 Due to the difficulty of describing UKF's as presented in the original formulations in a simple and systematized way, the forms of the UKF's shown in Table III are not necessarily the ones introduced by their corresponding authors. Nevertheless, the forms contained in this table, if different from the original ones, are trivial extensions (e.g., the additive form for the symmetric UKF of [7] in Table I is slightly more general). Besides, some of these extensions have already been explicitly proposed (e.g., the additive form of the symmetric UKF of [1] was modified in [31]). 
TABLE III

Most KNOWn Additive UnSCEnTEd KaLman Filters a

\begin{tabular}{|c|c|c|c|}
\hline & $\begin{array}{lll}\text { Pre. } & \text { Pos. } & \text { Pos. } \\
\text { sets }^{\text {b }} & \text { sets }^{\text {c }} & \text { CM }^{\text {d }}\end{array}$ & $\begin{array}{l}\text { Pos. } \\
\text { sets }^{\mathrm{c}}\end{array}$ & $\begin{array}{l}\text { Pos. } \\
\text { CMM }^{\text {d }}\end{array}$ \\
\hline \multirow[t]{2}{*}{1} & $\begin{array}{c}\text { Sym. UKF of [1] } \\
(N=2 n+1)\end{array}$ & \multicolumn{2}{|c|}{$\begin{array}{c}\text { Simp. UKF of [34] } \\
(N=n+1)\end{array}$} \\
\hline & Tab I [1] & Tab I [5] & (6) \\
\hline \multirow[t]{2}{*}{2} & $\begin{array}{c}\text { Sym. UKF of [7] } \\
(N=2 n+1)\end{array}$ & \multicolumn{2}{|c|}{$\begin{array}{c}\text { Min. UKF of [12] } \\
(N=n+1)\end{array}$} \\
\hline & Tab I [2] (10) (6) & Tab I [6] & (6) \\
\hline \multirow[t]{2}{*}{3} & $\begin{array}{l}\text { Red. UKF of [8] } \\
(N=n+1)\end{array}$ & \multicolumn{2}{|c|}{$\begin{array}{c}\text { Sc. UKF [38] } \\
(N \text { depends on the set })\end{array}$} \\
\hline & Tab I [3] & Tab II [1] Tab II [2] & Tab II [3] \\
\hline \multirow[t]{2}{*}{4} & $\begin{array}{c}\text { Sph. Simp. UKF of [9] } \\
(N=n+2)\end{array}$ & \multicolumn{2}{|c|}{$\begin{array}{l}\text { UKF with the AuxUT [38] } \\
(N \text { depends on the set })\end{array}$} \\
\hline & Tab I [4] & Tab II [4] Tab II [5] & Tab II [6] \\
\hline \multirow[t]{2}{*}{5} & $\begin{array}{c}\text { Sym. UKF of [35] } \\
(N=2 n+1)\end{array}$ & \multicolumn{2}{|c|}{$\begin{array}{c}\text { UKF of }[\mathbf{3 6}] \\
\left(N=2 n^{2}+1\right)\end{array}$} \\
\hline & Tab I [7] (10) (13) & Tab I [8] & (6) \\
\hline 6 & 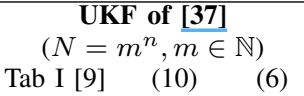 & -- & \\
\hline
\end{tabular}

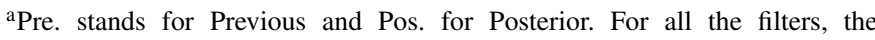
transformed means $\left(\hat{x}_{k \mid k-1}\right.$ and $\left.\hat{y}_{k \mid k-1}\right)$ are obtained by the left equation of (12), the CCM $\hat{P}_{x y}^{k \mid k-1}$ by (13), and the correction step is given by (7). ${ }^{\mathrm{b}}$ Points $\chi_{i}^{k-1 \mid k-1}, \chi_{i}^{k \mid k-1}$ and their weights. ${ }^{\mathrm{c} P o i n t s} \chi_{i}^{k \mid k-1}, \gamma_{i}^{k \mid k-1}$ and their weights. ${ }^{\mathrm{d}} \hat{P}_{x x}^{k \mid k-1}$ and $\hat{P}_{y y}^{k \mid k-1}$.

Remark 4 There are three other UKF's that are not presented in Table III: [40] describes a symmetric UKF matching up to the 4th central moment of the previous rv; [39], an asymmetric UKF matching up to the 3rd central moment of the previous $r v$; and [41], a symmetric UKF matching up to the 8th central moment of a scalar Gaussian rv. Table III does not show these UKF's because, instead of presenting their expressions, these works only show procedures from which these UKF's can be obtained.

Remark 5 Although some sigma sets can be composed by negative weights, one should note that these can lead to nonpositive sample CM's [7] and to higher errors [13].

There are applications where the machine precision is such that rounding errors can cause KF's to diverge. Hence, one should use algorithms that are less susceptible to such errors, which is the case of KF's in square-root form [42]. In order to propagate square-root matrices in square-root UKF's, one can use the $Q R$ decomposition and Cholesky update factor techniques (see [43]). To date, we are aware of five variants for the Square-Root Unscented Kalman Filters (SRUKF's): SRUKF of [43] (system in additive form (1) with the sigma set of [35], Tab I [7], and statistics calculation (10)-(13)); SRUKF of [30] (general form (2) with the set of [35]); SRUKF of [44] (additive form (1) with the spherical simplex set of [9], Tab I [4]); SRUKF of [4] (general form (2) with the set of [1], Tab I [1]); the Improved SRUKF of [45] (additive form (1) with the reduced set of [8], Tab I [3]).

Next, we present general comments about UKF variants and analyze some of their properties.

\section{B. Definitions for UKF's}

1) Variations on UKF definitions: From Section II-A, it is clear that there are many UKF variants. Given that, in general, these variants are not equivalent, one cannot properly point out which one is "the definition" for the UKF. Nonetheless, most works in the literature use the term 'UKF' when referring to either the UKF of [1] - as can be seen in [6], [19] - or to the UKF of [35] - as can be seen in [11], [46]. By comparing their sets of sigma points (cf. Tab I [1] with Tab I [7]), one can see that there are two main differences between these filters. First, the UKF of [1] uses a $\kappa$ to calculate the weights and the sigma points, while the one of [35] uses a term $\lambda=\alpha^{2}(n+\kappa)-n$ to do so. Second, in the UKF of [35], $w_{0}^{m}$ and $w_{0}^{c}$ are distinct objects, while in the UKF of [1], $w_{0}^{m}=w_{0}^{c}=w_{0}$.

2) Variation on scaled UKF definitions: Although the UKF of [35] (Tab III [5,1-3]) is described and widely referred to as a non-scaled UKF (cf. [11], [46]), Merwe himself, one of the authors in [35], describes this filter as a scaled UKF form (cf. [30]) - it has a scaling parameter $\alpha$ (cf. Tab I [7]). Apart from that, one should notice that this scaled UKF form differs from the ones proposed by [38] (the ones using the UT's of Table II).

\section{Accuracy of the UKF's}

1) Transformed covariance matrix: As [11] states, a large number of papers repeat the statement of [7] that the CM of transformed rv's is matched up to the second order in the UKF's when the mean and the CM of the prior rv are matched [7]. However, that is not true for all UKF variants. Indeed, [11] has already pointed out this issue for the symmetric UKF of [35] by providing a counter-example: considering $X \sim N\left(0_{n \times 1}, I_{n}\right)$ and $Y:=f(X)=X^{T} X$, the theoretical result for the CM of $Y$ is $P_{y y}=2 n$. The second order Taylor approximation gives the correct result, but the UT of [35] differs from it (see Table II in [11]).

2) Transformed cross covariance matrix: The transformed cross-covariance matrix (CCM) is necessary for the UKF, but the literature has not yet provided an estimation quality for it.

\section{Sigma sets composed by less than $2 n$ sigma points}

1) Reduced set of [8]: This set has two drawbacks. First, it can be numerically unstable for great values of $n$ due to the fact that the weights are composed by fractions of $2^{n}$ [9]. Second, neither the sample mean, $\mu_{\chi}$, nor the sample CM, $\Sigma_{\chi \chi}$, are equal to the mean and $\mathrm{CM}$ of the prior distribution when $n$ is greater than one [12]. In fact, from Tab I [3], for $n=2, X \sim\left([0]_{2 \times 1}, I_{2}\right)^{2}, w_{0}=0.5$, and using (11) with $w_{i}^{c}=w_{i}^{m}=w_{i}$, we have that $\mu_{\chi}=1 / 4[-1,3]^{T} \neq[0]_{2 \times 1}$ and $\Sigma_{\chi \chi}=\frac{1}{2}\left[\begin{array}{ll}1 & 1 \\ 1 & 5\end{array}\right] \neq I_{2}$.

2) Spherical simplex set of [9]: This set does not present the instability problem of the set of [8], but still has the same problem that neither $\mu_{\chi}$, nor $\Sigma_{\chi \chi}$, are equal to the mean and the CM of $X$ when $n$ is greater than one [12]. In fact, from Tab I [4], for $n=2, X \sim\left([0]_{2 \times 1}, I_{2}\right)^{2}, w_{0}=0.5$, and using (11) with $w_{i}^{c}=w_{i}^{m}=w_{i}$, we have that $\mu_{\chi}=1 /(2 \sqrt{6})[0,1]^{T} \neq$ $[0]_{2 \times 1}$ and $\Sigma_{\chi \chi}=\operatorname{diag}(1,53 / 96) \neq I_{2}$.

3) Minimum set of [34]: The CM for this set is not matched. Using Tab I [5] and (11), it can be shown that $\Sigma_{\chi \chi}=P_{X X}+1 /(n+1)[\bar{X}]_{1 \times(n+1)}\left[\bar{X}^{T}\right]_{(n+1) \times 1}$ for $X \sim\left(\bar{X}, P_{X X}\right)^{n}$. In fact, for $X \sim(3,4)^{1}, \Sigma_{\chi \chi}=13 \neq 4$. 
4) Minimum set of [12]: This is the only sigma set composed by less than $2 n$ points matching the mean and $\mathrm{CM}$ of $X$.

\section{E. Scaled Transformations}

1) Scalable sigma sets: the authors in [38] state that the "scaled unscented transform [...] allows any set of sigma points to be scaled by an arbitrary scaling factor" (the italic is of the authors and the bold is ours). However, suppose $X \sim$ $\left([0]_{2 \times 1}, I_{2}\right)^{2}$ and that the previous sigma set is $\chi_{1}=[\sqrt{2}, 0]^{T}$, $\chi_{2}=\left[0, \sqrt{2}^{T}, \chi_{3}=[-\sqrt{2}, 0]^{T}, \chi_{4}=[0,-\sqrt{2}]^{T}\right.$ and $w_{1}=w_{2}=w_{3}=w_{4}=\frac{1}{4}$. From Tab II [1:3], one can see that, for $\alpha=0.5$ and $\chi_{1}^{\prime}=\chi_{1}=\left[\begin{array}{ll}0 & 2\end{array}\right]^{T}$, the sample mean, $\mu_{\chi^{\prime}}$, and the sample $\mathrm{CM}, \Sigma_{\chi^{\prime}}$, of the (previous) scaled sigma set are $\mu_{\chi^{\prime}}=[-\sqrt{2}, 0]^{T} \neq[0]_{2 \times 1}$ and $\Sigma_{\chi^{\prime}}=\operatorname{diag}\left([-3,1]^{T}\right) \neq I_{2}$.

This example shows that the sample mean and the sample $\mathrm{CM}$ of $\chi^{\prime}$ are not equal to the mean and CM of $X$, respectively. In fact, as one can see from the following theorem, this property is not guaranteed to hold for any sigma set, except for those having one sigma point equal to the mean of $X$.

Theorem 1 Consider $X \sim\left(\bar{X}, P_{X X}\right)^{n}$ and a function $f$ : $\mathbb{R}^{n} \rightarrow \mathbb{R}^{n_{y}}$ defining a new $r v Y:=f(X)$ and consider a set of sigma points $\chi=\left\{\chi_{i}, w_{i}\right\}_{i=1}^{N}$ for $X$. Consider also the set of scaled sigma points $\chi^{\prime}=\left\{\chi_{i}^{\prime}, w_{i}^{\prime}\right\}_{i=1}^{N}$ obtained from the scaled UT of [38] (Tab II [1:3]). We have that:

1) if $\sum_{i=1}^{N} w_{i}=1$, then $\sum_{i=1}^{N} w_{i}^{\prime}=1$;

2) $\mu_{\chi^{\prime}}=\frac{1}{\alpha} \mu_{\chi}+\chi_{1}\left(1-\frac{1}{\alpha}\right)$;

3) if $\chi_{1}=\bar{X}$, then $\mu_{\chi^{\prime}}=\mu_{\chi}$;

4) if $\alpha \neq 1$, then $\chi_{1}=\bar{X} \Leftrightarrow \mu_{\chi^{\prime}}=\mu_{\chi}$;

5) if $\chi_{1}=\bar{X}$, then $\Sigma_{\chi^{\prime} \chi^{\prime}}=\Sigma_{\chi \chi}$.

Proof: Suppose $\sum_{i=1}^{N} w_{i}=1$, then $\sum_{i=1}^{N} w_{i}^{\prime}=\frac{1}{\alpha^{2}}(-$ $\left.1+\alpha^{2}+\sum_{i=1}^{N} w_{i}\right)=1$. For the second and third assertion, $\mu_{\chi^{\prime}}=\frac{1}{\alpha} \mu_{\chi}+\chi_{1}\left(1-\frac{1}{\alpha}\right)$, which, supposing $\chi_{1}=\mu_{\chi}$, gives $\mu_{\chi^{\prime}}=\mu_{\chi}$ and, supposing $\mu_{\chi^{\prime}}=\mu_{\chi}, \alpha \neq 1$, gives $\chi_{1}=\mu_{\chi}$. The last assertions can be proven by the fact that $\Sigma_{\chi^{\prime} \chi^{\prime}}=$ $w_{1}^{\prime}\left(\chi_{1}^{\prime}-\mu_{\chi}\right)(\diamond)^{T}+\sum_{i=2}^{N} w_{i}^{\prime}\left(\chi_{1}+\alpha\left(\chi_{i}-\chi_{1}\right)-\mu_{\chi}\right)(\diamond)^{T}$, which, for $\chi_{1}=\mu_{\chi}$, gives $\Sigma_{\chi^{\prime} \chi^{\prime}}=\Sigma_{\chi \chi}$.

Therefore, the scaled UT of [38] is restrictive in the sense that this UT does not provide the mentioned results for any previous set of sigma points. On the other hand, this restriction is not applied to the AuxUT of [38].

2) Covariance matrix: Consider $X \sim N\left([0]_{3 \times 1}, I_{3}\right)^{3}$ and $Y:=f(X)=X^{T} X$. Then, $\bar{Y}=3$ and $P_{Y Y}=6$. Using the scaled UT of [38] with the symmetric sigma set of [7], we get, from (12), $\mu_{\gamma}=3=\bar{Y}$ and $\Sigma_{\gamma \gamma}^{*}=3 \alpha^{2}-8 \neq P_{Y Y}$. This result shows two problems involving the matching of the CM. First, the transformed CM for this scaled UT is not matched up to the order 2, but only to the order 1 . Second, the scaling factor modifies the $\mathrm{CM}$ even for second order polynomial approximation.

3) Cross-covariance matrix: Similar to the case for the non-scaled UTs, the estimation quality of CCM's for the scaled UT of [38] and for the AuxUT of [38] has not been presented in the literature yet. Besides, there is no mention of the influence of the scaling factor on the transformed CCM for the UKF of [35]. Since it is desirable to match the first and the second moments, the free parameter $\alpha$ should modify only the third and higher terms. However, consider $X \sim N\left(0_{3 \times 1}, I_{3}\right)$ and $Y:=f(X)=X^{T} X$. Then, from Tab I [7], (12) and (13), we have $\mu_{\gamma}=3=\bar{Y}$ and $\Sigma_{\chi \gamma}=6 \alpha\left[I_{3}\right]_{* i}-\frac{9}{2 \alpha}\left[I_{3}\right]_{* i}$. Therefore, the second order term is also modified.

\section{F. Square-root forms of the UKF's}

1) Downdating the Cholesky factor: For an equation in the form $A A^{T}=R R^{T}-S S^{T}$, where $A, R, S$ are Cholesky factors, we say that $A$ is a downdated Cholesky factor of $R$ by $S$. There are three parts within the SRUKF algorithms in the literature where Cholesky factors are downdated: in the calculations of the square-root matrices (SRM's) of the predicted state's CM, of the innovation's CM, and of the corrected state's CM. In the first two steps, the downdating steps are performed only for the sigma points with negative weights, while, in the last, they are always performed.

Since the direct downdating of a Cholesky factor is "inherently more ill-conditioned than if $Q$ (the $Q$ matrix of a $Q R$ decomposition) is also available" [47] (the comment within parentheses is ours), filters resulting from the substitution of downdating steps by $Q R$ decompositions - or, more generally, by any triangulation technique [6] - should be computationally more stable. In fact, [48] has developed such a technique for calculating the SRM of the corrected state's CM for quadrature Kalman filters and [6] for the CKF.

2) Square-Root Scaled UKF: The literature does not present any filter conjugating the SRUKF with the scaled UT of [38] (Tab II [1:3]) nor with the AuxUT (Tab II [4:6]).

3) Square-Root UT: Although there are definitions for SRUKF's, we have not been able to find any definition for a Square-Root Unscented Transformation (SRUT). The importance of defining a SRUT can be motivated by the importance of defining an UT: it gives its resulting UKF a better mathematical formal principle; it is possible to study a UKF focusing on its respective UT, since it is the core difference between the UKF relative to other nonlinear KFbased filters; one can apply a UT not only within the KF framework, but in any framework or application that requires uncertainty propagation (e.g. [49]) or within other stochastic filter (e.g. [50]).

\section{G. Conclusion of the literature review}

This section showed that many variations for the UKF have been proposed. Some of them are different in the recursive filter framework, others in the composition of sigma sets, and others in the transformation from the previous to the posterior sigma sets. Sections II-B to II-F presented some problems and gaps within the UKF theory.

The difficulty in gathering all results related to Unscented and the problems within some of them reveal a lack of foundation in terms of mathematical principles and also the absence of mathematical solutions generalizing the sigma sets, UTs and UKFs of the literature. In order to address these needs, we propose a systematization that treats the construction of UKF's in parts. We first consider the problem of estimating the mean of a non-linear transformation, which will lead us to the definition of the sigma-representation. 


\section{SigMA-REPRESENTATIONS}

Given a rv $X \in \Phi^{n}$ with pdf $p_{X}(x)$, many problems, such as calculating the moments of an rv, can be reduced to the problem of finding the posterior expectation

$$
E\{f(X)\}=\int_{\mathbb{R}^{n}} f(\xi) p_{X}(\xi) d \xi,
$$

for an appropriate function $f: \mathbb{R}^{n} \rightarrow \mathbb{R}^{n_{y}}$. As a first attempt to solve this problem, one could consider using numerical integration techniques. In the scalar case $\left(n=n_{y}=1\right)$ and if the function $f$ is well approximated by a polynomial of order $2 N-1$, the Gaussian quadrature methods give approximate solutions for (14) of the form (see [5], [51]-[54])

$$
E\{f(X)\}=\int_{-\infty}^{\infty} f(\xi) p_{X}(\xi) d \xi \approx \sum_{i=1}^{N} w_{i} f\left(x_{i}\right)
$$

where $x_{1}, \ldots, x_{N}$ are samples of $X$. For $X$ being a standard scalar normal rv, the solution is obtained by the Gauss-Hermite Quadrature (GHQ) [5], [30], [51]-[53], [55]. The multivariate case can be obtained by first using a stochastic decoupling technique $X^{\prime}={\sqrt{P_{X X}}}^{-1}(X-\bar{X})$, where $X^{\prime}$ is a multivariate standard Gaussian rv. Then, for $\tilde{f}(X):=f\left({\sqrt{P_{X X}}}^{T} X+\bar{X}\right)$, the GHQ is applied on the form

$$
\begin{aligned}
E_{X^{\prime}}\left\{\tilde{f}\left(X^{\prime}\right)\right\}= & \int_{\mathbb{R}^{n}} \tilde{f}(\xi) p_{X^{\prime}}(\xi) d \xi \\
& \approx \sum_{i_{1}, \ldots, i_{n}=1}^{N} w_{i_{1}} \cdots w_{i_{n}} \tilde{f}\left(x_{i_{1}}, \ldots, x_{i_{n}}\right),
\end{aligned}
$$

and $E_{X}\{f(X)\}$ is obtained from $E_{X^{\prime}}\left\{\tilde{f}\left(X^{\prime}\right)\right\}$ [5]. An alternative to solving the multivariate Gaussian case is to use the spherical cubature rule along with the Gaussian Quadrature after performing a Cartesian-to-spherical coordinate transformation. In fact, consider the Gaussian case $p_{\xi}(\xi)=\exp \left(-\xi \xi^{T}\right)$ and let $\xi=\rho y$, with $y^{T} y=1, \rho \in[0, \infty)$. In this case, (14) becomes

$$
E\{f(X)\}=\int_{0}^{\infty} S(\rho) \rho^{n-1} \exp \left(-r^{2}\right) d \rho, S(\rho):=\int_{U_{n}} f(\rho y) d \phi(y),
$$

where $U_{n}:=\left\{u \in \mathbb{R}^{n} \mid u^{T} u=1\right\}$ and $\phi(\bullet)$ is the spherical surface measure of $U_{n}$ [6]. The spherical integral $S(\rho)$ is solved by the spherical cubature rule, while the expectation by a Gaussian Quadrature rule [6].

Instead of using a quadrature solution, one can obtain a suboptimal solution by approximating the function $f$. For instance, one can use linearization or higher-order polynomial approximations of the kind $f(x) \approx \sum_{k} a_{k} x^{k}$ [56]. In this case, (14) would be approximated by $E\{f(X)\}=$ $\sum_{k} a_{k} \int_{\mathbb{R}^{n}} \xi^{k} p_{X}(\xi) d \xi$. Well-known methods are the trapezoidal rule, Simpson's Rule, the Newton-Cotes Formulas, the Clenshaw-Curtis Integration, among others [56].

Another alternative for obtaining (14) is by approximating $p_{X}(x)$. We can classify this type of suboptimal approximation into two categories, namely Monte Carlo methods [23]-[27], [57] and sigma-point methods [30], [55]. Monte Carlo (MC) methods consist of taking a very large quantity of samples $x_{i}$ of $X$ (the method gets more accurate as the number of samples $N \rightarrow+\infty$ ) randomly [23], [24], [26], [27]. Sigma point methods, on the other hand, consist on analytically choosing finite $N$ samples $x_{i}$ and weights $w_{i}$ [30]. These approaches can be viewed as generalized-negative weights are admitteddiscrete approximations of $p_{X}(x)$.

There is some overlap in this type of classification, as well as other interpretations. Some sigma-point formulas can be obtained from integration approaches [15], [33], [58]. For instance, [6] derives a particular case of the symmetric sigmapoint set of [7] (Tab I [2]) using the spherical cubature quadrature; and [36] and [13] derive the fifth-order sigma-point set (Tab I [8]) also by this quadrature rule [15]. It is worthwhile to mention that the symmetric sigma-point set of [7] can also be viewed as a statistical linear regression technique [31].

All techniques for expected value calculation can be used for dynamical systems (1) or (2) in order to obtain recursive filters. For instance, GHQ yields the GHF [5] when applied in a $\mathrm{KF}$ framework; the cubature spherical rule yields the CKF [6], [29]; the Central Difference technique, the CDF [5]; the linearization and the second order approximation of the functions yield the EKF and the SOEKF, respectively; different UT's yield different forms of the UKF; Stirling's interpolation formula yields the Divided Difference Filter (DDF) [28]; and the Monte Carlo methods yield SMCF's (e.g. PF's [23]-[26]) or MCMCF's [27].

The DDF and the CDF are considered to be "essentially identical" [30]. The CKF is a particular case of the derivations in [36] and [13], where the CKF is also showed to be equivalent to the UKF of [1] (Tab III [1,1:3]) by making the central weight equal to zero [11], [19].

The UKF of [1] is showed to be a particular case of the GHF in the scalar case $\left(n=n_{y}=1\right)$ [5]. In fact, consider a scalar standard normal rv $X \sim N(0,1)$. Both a GHQ approach of order $N=3$ and a sigma set of [1] with $k=2$ and $n=1$ would yield the set $\chi_{1}=-\sqrt{3}, \chi_{2}=0, \chi_{3}=\sqrt{3}, w_{1}=$ $w_{3}=\frac{1}{6}, w_{2}=\frac{2}{3}$ [5]. However, for larger lengths of the state vector, this equivalence does not hold. The GHF is $\mathcal{O}\left(N^{n}\right)$, while the UKF of [1] is $\mathcal{O}\left(n^{3}\right)$ [5], [13], [55]. In fact, for $X \sim\left([0]_{2 \times 1}^{T}, I_{2}\right)^{2}$, the Gauss-Hermite set would be $w_{i}=$ $1 / 36, i=1,3,7,9 ; w_{j}=1 / 9, j=2,4,6,8 ; w_{5}=4 / 9 ; \chi_{1}=$ $\chi_{3}=-\chi_{7}=-\chi_{9}=[\sqrt{3}, \sqrt{3}]^{T} ; \chi_{2}=-\chi_{8}=[0, \sqrt{3}]^{T} ; \chi_{4}=$ $-\chi_{6}=[\sqrt{3}, 0]^{T} ; \chi_{5}=[0]_{2 \times 1}$; while the sigma set of [1] (Tab I [1]) for $k=2$ and $n=2$ would be $w_{0}=0.5 ; w_{1}=\ldots=$ $w_{4}=0.125 ;$ and $x_{0}=[0,0]^{T} ; x_{1}=-x_{3}=[2,0]^{T} ; x_{2}=$ $-x_{4}=[0,2]^{T}$.

In order to properly construct the systematization of the UKF filtering theory, we propose definitions of three fundamental mathematical elements: (I) the sigma $(\sigma)$ representation; (II) the Unscented Transformation; and (III) the recursive filters. The first is an approximation of a rv's pdf by a set of weighted points. The second is an approximation of the joint pdf of two rv's by two sets of weighted points, where one is a function of the other. The third consists of solutions to the stochastic filtering problems applying the UT in a recursive manner.

\section{A. Sigma-representation}

The $\sigma$-representations ( $\sigma$ R's) are approximations of a rv's pdf by a set of weighted points via moment matching. We say 
that a set is an $l$ th order $\sigma \mathrm{R}$ of a rv if the central moments of its samples are equal to the central moments of the chosen rv up to, and including, order $l$.

The case $l=2$ is of particular interest, since the majority of works in Unscented literature focus on second order moment matching [1]-[4], [7]-[9], [30], [38], [40], [43], [59]. This is mainly motivated by three facts. First, these are usually the estimated statistics within a stochastic filter. Second, they fully describe a Gaussian distribution [55]. Third, the mean is the point estimate with the least mean squared error.

The notation $M_{X}^{j}$ stands for the $j$ th central moment of $X \in$ $\Phi^{n}$, and is defined, for even and odd $j$, respectively, as

$$
\begin{aligned}
& M_{X}^{j}:=E\left\{\left[(X-\bar{X})(X-\bar{X})^{T}\right]^{\otimes \frac{j}{2}}\right\}, \\
& M_{X}^{j}:=E\left\{\left[(X-\bar{X})(X-\bar{X})^{T}\right]^{\otimes \frac{j-1}{2}} \otimes(X-\bar{X})\right\} .
\end{aligned}
$$

Definition 1 (Sigma $(\sigma)$-Representation) Define, for even and odd $j$, respectively,

$$
\begin{aligned}
\mathcal{M}_{\chi}^{j} & :=\sum_{i=1}^{N} w_{i}^{(j)}\left[\left(\chi_{i}-\mu_{\chi}\right)\left(\chi_{i}-\mu_{\chi}\right)^{T}\right]^{\otimes \frac{j}{2}}, \\
\mathcal{M}_{\chi}^{j} & :=\sum_{i=1}^{N} w_{i}^{(j)}\left[\left(\chi_{i}-\mu_{\chi}\right)\left(\chi_{i}-\mu_{\chi}\right)^{T}\right]^{\otimes \frac{j-1}{2}} \otimes\left(\chi_{i}-\mu_{\chi}\right),
\end{aligned}
$$

as the jth sample central moment of $\chi:=\left\{\chi_{i}, w_{i}^{(1)}, \ldots\right.$, $\left.w_{i}^{(l)}\right\}_{i=1}^{N}$, which has sample mean $\mu_{\chi}:=\sum_{i=1}^{N} w_{i}^{(1)} \chi_{i}$ and consider $X \sim\left(\bar{X}, M_{X}^{2}, \ldots, M_{X}^{l}\right)^{n}$. Then $\chi$ is an lth order $\mathbf{N}$ points $\sigma$-representation (lth $\mathrm{N} \sigma \boldsymbol{R})$ of $X$ if

$$
\begin{aligned}
& w_{i}^{(j)} \neq 0, i=1, \ldots, N \text { and } j=1, \ldots, l \\
& \mu_{\chi}=\bar{X} ; \quad \mathcal{M}_{\chi}^{j}=M_{X}^{j}, j=2,3, \ldots, l .
\end{aligned}
$$

Besides, assume $\chi$ to be an lthN $\sigma R$ of $X$, then:

- $\chi$ is normalized if $\sum_{i=1}^{N} w_{i}{ }^{(j)}=1, \quad j=1,2, \ldots, l$.

- $\chi$ is symmetric if, for $N$ odd, $\chi_{i}-\chi_{N}=-\left(\chi_{i+\frac{N-1}{2}}-\right.$ $\left.\chi_{N}\right)$ and $w_{i}^{(j)}=w_{i+\frac{N-1}{2}}^{(j)}, 1 \leq i \leq \frac{N-1}{2}$; or for $N$ even, $\chi_{i}-\chi_{N}=-\left(\chi_{i+\frac{N}{2}}-\chi_{N}\right)$ and $w_{i}^{(j)}=w_{i+\frac{N}{2}}^{(j)}$, $1 \leq i \leq \frac{N}{2}$.

- $\chi$ is homogeneous if, for $N$ odd, $w_{1}^{(j)}=w_{i}^{(j)}, 1 \leq i \leq$ $N-1$, or for $N$ even, $w_{1}^{(j)}=w_{i}^{(j)}, 1 \leq i \leq N$

When calling an $l$ th $N \sigma \mathrm{R}$ of $X$, the reference to the $l$ th order can be omitted if $l=2$. Also, the reference to $N$ point and/or to $X$ can be omitted in case they are obvious from the context or irrelevant for a given statement.

Theorem $2 X \sim\left(\bar{X}, M_{X}^{2}, \ldots, M_{X}^{l}\right)^{n}$ admits a normalized lthN $\sigma R$ if and only if there exists $E \in \mathbb{R}^{n \times N}, w^{(1)}:=$ $\left[w_{1}^{(1)}, \ldots, w_{N}^{(1)}\right]^{T}, W^{(j)}:=\operatorname{diag}\left(w^{(j)}\right)$, where $w^{(j)}:=$ $\left[w_{1}^{(j)}, \ldots, w_{N}^{(j)}\right]^{T}$, for $j=2,3, \ldots, l$ that satisfy, for $l$ even, the following equations:

$$
\begin{aligned}
& {\left[E_{* 1}^{\otimes \frac{j}{2}}, \ldots, E_{* N}^{\otimes \frac{j}{2}}\right] W^{(j)}\left[E_{* 1}^{\otimes \frac{j}{2}}, \ldots, E_{* N}^{\otimes \frac{j}{2}}\right]^{T}=M_{X}^{j}, j=2,4, \ldots, l ;} \\
& \quad\left[E_{* 1}^{\otimes \frac{j+1}{2}}, \ldots, E_{* N}^{\otimes \frac{j+1}{2}}\right] W^{(j)}\left[E_{* 1}^{\otimes \frac{j-1}{2}}, \ldots, E_{* N}^{\otimes \frac{j-1}{2}}\right]^{T} \\
& \quad=M_{X}^{j}, j=1,3, \ldots, l-1 ;
\end{aligned}
$$

$$
\begin{aligned}
E w^{(1)} & =0 ; \\
{[1]_{1 \times N} w^{(j)} } & =1, \quad j=1,2, \ldots, l .
\end{aligned}
$$

If (15) - (18) admits a solution $\left(E, w^{(1)}, W^{(2)}, \ldots, W^{(l)}\right)$, then a normalized lth order lthNoR of $X$ is $\left\{\chi_{i}, w_{i}^{(1)}, \ldots, w_{i}^{(l)}\right\}_{i=1}^{N}$ such that $\left[\chi_{1}, \ldots, \chi_{N}\right]:=E+[\bar{X}]_{1 \times N}$.

Proof: Define $E:=\left[\chi_{1}-\mu_{\chi}, \ldots, \chi_{N}-\mu_{\chi}\right]$. So, from the definition of $\mathcal{M}_{\chi}^{j}$, for $j$ even,

$$
\begin{aligned}
\mathcal{M}_{\chi}^{j} & =\sum_{i=1}^{N}\left[E_{* i}^{\otimes \frac{j}{2}}\right] w_{i}^{(j)}\left[E_{* i}^{\otimes \frac{j}{2}}\right]^{T} \\
& =\left[E_{* 1}^{\otimes \frac{j}{2}}, \ldots, E_{* N}^{\otimes \frac{j}{2}}\right] W^{(j)}\left[E_{* 1}^{\otimes \frac{j}{2}}, \ldots, E_{* N}^{\otimes \frac{j}{2}}\right]^{T},
\end{aligned}
$$

which proves (15). (16) can be proven similarly and the remaining is trivial.

Results for odd $l$ can be obtained in a similar fashion. The next corollary uses Theorem 2 to obtain two novel results: the minimum amount of $\sigma$-points for both the symmetric and the non-symmetric case when $P_{X X} \geq 0$. Note that the previouslystated result that the minimum number is $n+1$ for $P_{X X}>0$ [7]-[9] is a particular case of Corollary 1.

Corollary 1 Let $\chi:=\left\{\chi_{i}, w_{i}^{(1)}, \ldots, w_{i}^{(l)}\right\}_{i=1}^{N}$ be an lth $N \sigma R$ of $X \in \Phi^{n}$, which has $C M P_{X X}$ with rank $r \leq n$. Then

1) $N \geq r+1$. If $N=r+1$, then $\chi$ is a minimum lth $N \sigma R$ of $X$.

2) If $\chi$ is symmetric, then $N \geq 2 r$. In this case, if $N=2 r$, then $\chi$ is a minimum symmetric lth $N \sigma R$ of $X$.

Proof: To prove assertion 1), consider, first, $E \in \mathbb{R}^{n \times N}$ and the singular value decomposition of $P_{X X}, P_{X X}=$ $U S V^{T}$, where $S:=\operatorname{diag}\left(\left[\alpha_{1}, \ldots, \alpha_{r},[0]_{(n-r) \times 1}\right]^{T}\right)$, and $\alpha_{1}, \ldots, \alpha_{r}$ are the singular values of $P_{X X}$. Assume, for contradiction, $m:=\operatorname{rank}\{E\}<r$. Then there exists $v:=$ $\left[v_{1}^{T},[0]_{1 \times(n-r)}^{T}\right]^{T}, v_{1} \in \mathbb{R}^{r}, v_{1} \neq 0$, such that $v^{T} E=0$. Then, from (15),

$$
E W^{(2)} E^{T}=\left[\begin{array}{cc}
U_{1} & U_{2} \\
U_{3} & U_{4}
\end{array}\right] S V^{T} \Leftrightarrow v_{1}^{T} U_{1} S_{1}=0 \Leftrightarrow v_{1}^{T}=0,
$$

which is a contradiction. Therefore, $m \geq r$. Second, suppose $N=m$. Then, $E$ is full column rank and, from (17), $w^{(1)}=0$, which is a contradiction for, from Definition $1, w^{(1)} \neq 0$. So $N \geq \operatorname{rank}\{E\}+1 \geq r+1$. To prove assertion 2 ), let $\chi$ be symmetric. Then $E=\left[E_{2},-E_{2}\right]$, where $E_{2} \in \mathbb{R}^{n \times \frac{N}{2}}$. So

$r \leq \operatorname{rank}\{E\}=\operatorname{rank}\left\{\left[E_{2},-E_{2}\right]\right\}=\min \left\{n, \frac{N}{2}\right\} \Leftrightarrow N \geq 2 r$.

Corollary 2 Let $\chi=\left\{\chi_{i}, w_{i}^{(1)}, \ldots, w_{i}^{(l)}\right\}_{i=1}^{N}$ be a normalized lthN $\sigma R$ of $X \sim\left(\bar{X}, M_{X}^{2}, \ldots, M_{X}^{l}\right)^{n}$ and consider the $r v Z=A X+b, A \in \mathbb{R}^{n \times n}, b \in \mathbb{R}^{n}$. Then, the set $\Xi:=\left\{\xi_{i}, w_{i}^{(1)}, \ldots, w_{i}^{(l)} \mid \xi_{i}=A \chi_{i}+b\right\}_{i=1}^{N}$ is a normalized lthN $\sigma R$ of $Z$. In particular, we have $\mu_{\xi}=A \bar{X}+b$ and $\Sigma_{\xi \xi}=A P_{X X} A^{T}$.

Proof: The sample mean of $\Xi$ is $\mu_{\xi}=$ $\sum_{i=1}^{N} w_{i}^{(1)}\left(A \chi_{i}+b\right)=\bar{Z}$. The $j$-th sample central moment of $\Xi, j=2,4, \ldots, l$ ( $l$ even $)$ is

$M_{\xi}^{j}=A^{\otimes \frac{j}{2}}\left[\sum_{i=1}^{N} w_{i}^{(j)}\left[\left(\chi_{i}-\mu_{\chi}\right) \otimes\left(\chi_{i}-\mu_{\chi}\right)^{T}\right]^{\otimes \frac{j}{2}}\right]\left(A^{T}\right)^{\otimes \frac{j}{2}}$ 


$$
=A^{\otimes \frac{j}{2}} M_{X}^{j}\left(A^{T}\right)^{\otimes \frac{j}{2}}=M_{Z}^{j} .
$$

The odd $j$ case can be similarly proven.

The result used by [7], [40], [59] and others that a sigma set $\left\{\chi_{i}, w_{i}\right\}_{i=1}^{N}$ approximating an $\mathrm{rv} X \sim\left(\bar{X}, P_{X X}\right)^{n}$ can be obtained by the transformation $\chi_{i}=\sqrt{P_{X X}} \xi_{i}+\bar{X}$, where $\left\{\xi_{i}, w_{i}\right\}_{i=1}^{N}$ is a sigma set of an rv with mean $[0]_{n \times 1}$ and covariance matrix $I_{n}$, is a particular case of Corollary 2 .

\section{B. Particular sigma-representations}

Motivated by Theorem 2 and Corollary 1, we now seek a $\sigma \mathrm{R}$ for the minimum symmetric and the minimum cases.

1) Minimum Symmetric $\sigma$-Representation: Let $\chi=$ $\left\{\chi_{i}, w_{i}^{m}, w_{i}^{c}\right\}_{i=1}^{2 n}$ be a $\sigma \mathrm{R}$ of $X \sim\left(\bar{X}, P_{X X}\right)^{n}, P_{X X} \geq$ 0 . Considering the equations of Theorem 2 , suppose $\chi$ is minimum symmetric. Then, we have $E=[\bar{E},-\bar{E}]$, where $\bar{E} \in \mathbb{R}^{n \times n}$. Define $W:=\operatorname{diag}\left(\left[w_{1}^{c} \cdots w_{n}^{c}\right]^{T}\right)>0$, Then, from (15),

$$
([\bar{E},-\bar{E}] \sqrt{\operatorname{diag}([W, W])})(\diamond)^{T}=2^{-1}\left(\sqrt{P_{X X}}\left[I_{n},-I_{n}\right]\right)(\diamond)^{T} .
$$

Clearly, a sufficient condition is $\bar{E}=(\sqrt{2 W})^{-1} \sqrt{P_{X X}}$. Since (17) is satisfied for all symmetric $\sigma \mathrm{R}$ 's, a closed form for this case is obtained. The next corollary formalizes it.

Corollary 3 (Minimum Symmetric $\boldsymbol{\sigma}$-representation) Consider a symmetric $X \sim\left(\bar{X}, P_{X X}\right)^{n}, P_{X X}>0 . A$ minimum symmetric $\sigma R$ of $X$ is the set $\chi=\left\{\chi_{i}, w_{i}^{m}, w_{i}^{c}\right\}_{i=1}^{2 n}$ with $W:=\operatorname{diag}\left(\left[w_{1}^{c} \cdots w_{n}^{c}\right]^{T}\right)>0, w_{i}^{m} \neq 0$ and

$$
\begin{aligned}
& {\left[\chi_{1} \cdots \chi_{2 n+1}\right]=} \\
& {\left[(\sqrt{2 W})^{-1} \sqrt{P_{X X}},-(\sqrt{2 W})^{-1} \sqrt{P_{X X}}\right]+[\bar{X}]_{1 \times 2 n} .}
\end{aligned}
$$

In addition, if $\sum_{i=1}^{2 n} w_{i}^{m}=\sum_{i=1}^{2 n} w_{i}^{c}=1$, then $\chi$ is normalized. Moreover, if $W=(1 /(2 n)) I_{n}$ and $w_{i}:=w_{i}^{m}=w_{i}^{c}$, $i=1, \ldots, 2 n$, then $\chi=\left\{\chi_{i}, w_{i}\right\}_{i=1}^{2 n}$ is a (normalized) homogeneous minimum symmetric $\sigma$-representation of $\mathrm{X}$.

If an extra point located on $\bar{X}$ is added to this $\sigma \mathrm{R}$, neither the sample mean, nor the sample CM, will be modified. Therefore, the extra point's weight can act as a tuning parameter.

\section{Corollary 4 ([Odd] Min. Symmetric $\boldsymbol{\sigma}$-representation)}

Consider a symmetric $X \sim\left(\bar{X}, P_{X X}\right)^{n}, P_{X X} \geq 0$. An odd minimum symmetric $\sigma R$ of $X$ is the set $\chi=\left\{\chi_{i}, w_{i}^{m}, w_{i}^{c}\right\}_{i=1}^{2 n}$ with $W:=\operatorname{diag}\left(\left[w_{1}^{c} \cdots w_{n}^{c}\right]^{T}\right)>0, w_{i}^{m} \neq 0$, and

$$
\begin{aligned}
& {\left[\chi_{1} \cdots \chi_{2 n+1}\right]=} \\
& {\left[(\sqrt{2 W})^{-1} \sqrt{P_{X X}},-(\sqrt{2 W})^{-1} \sqrt{P_{X X}}, 0_{n \times 1}\right]+[\bar{X}]_{1 \times(2 n+1)} .}
\end{aligned}
$$

In addition, if $\sum_{i=1}^{2 n+1} w_{i}^{m}=\sum_{i=1}^{2 n+1} w_{i}^{c}=1$, then $\chi$ is a normalized odd minimum symmetric $\sigma$-representation (MiSy $\sigma$ R). Moreover, if $W=\left(\left(1-w_{2 n+1}\right) /(2 n)\right) I_{n}$ and $w_{i}:=w_{i}^{m}=w_{i}^{c}$, $i=1, \ldots, 2 n+1$, then $\chi=\left\{\chi_{i}, w_{i}\right\}_{i=1}^{2 n+1}$ is a (normalized) homogeneous (odd) minimum symmetric $\sigma$-representation (HoMiSy $\sigma \boldsymbol{R})$ of $\mathbf{X}$, which is equivalent to the symmetric sigma set of [7] (Tab I [2]).

Corollaries 3 and 4 present the even and odd $\sigma$ R's with the least amount of symmetric sigma points. The classical symmetric sigma sets of [1], [7] (Table I), which have been presented in the literature without formal justification, are rewritten forms of the homogeneous cases of these corollaries.

Regarding the choice of the tuning parameter, a couple of results have already been proposed in the literature. The authors in [60] provide an off-line way of computing it by maximizing the likelihood function with a training set of data. In [19], an on-line method of computing the tuning parameter by means of maximizing a Gaussian approximation of the likelihood function is proposed.

2) Minimum Sigma-Representation: In this section, we look for a $\sigma \mathrm{R}$ with the least amount of (non-symmetric) sigma points. For $P_{X X}>0$, this number is $n+1$ (cf. Corollary 1). We first present a heuristic for finding this $\sigma \mathrm{R}$, followed by a formal and more general minimum $\sigma \mathrm{R}(\mathrm{Mi} \sigma \mathrm{R})$ in Theorem 3 . At the end of this section, Corollary 5 shows that this minimum $\sigma \mathrm{R}$ has the minimum sigma set of [12] as a particular case.

Let $\left\{\chi_{i}, w_{i}\right\}_{i=1}^{n+1}$ be a normalized minimum $\sigma \mathrm{R}$ of $X \sim$ $\left(\bar{X}, P_{X X}\right)^{n}, P_{X X}>0$. From Theorem 2 and Corollary 1, $E=[\bar{E}, e]$, where $\bar{E} \in \mathbb{R}^{n \times n}$ and $e \in \mathbb{R}^{n}$. Define $\bar{w}=\left[w_{1} \ldots w_{n}\right]^{T}$ and $\bar{W}:=\operatorname{diag}(\bar{w})>0$. Then, from (15), $e=-w_{n+1}^{-1} \bar{E} \bar{w}$. Substituting it on (17),

$$
\begin{aligned}
P_{X X} & =\bar{E} \bar{W} \bar{E}^{T}+w_{n+1}^{-1} \bar{E} \bar{w} \bar{w}^{T} \bar{E}^{T} \\
& =\bar{E} \sqrt{\bar{W}}\left(I_{n}+v v^{T}\right)(\bar{E} \sqrt{\bar{W}})^{T},
\end{aligned}
$$

where $v:=w_{n+1}^{-\frac{1}{2}} \sqrt{\bar{W}} \bar{w}$. Then $\bar{E}=\sqrt{P_{X X}}\left(I_{n}+\right.$ $\left.v v^{T}\right)^{-\frac{1}{2}} \bar{W}^{-\frac{1}{2}}$ is a sufficient condition. Moreover, from (17), $w_{n+1}=1 /\left(1+\sum_{i=1}^{n} v_{i}^{2}\right)$.

The following theorem gives the minimum $\sigma \mathrm{R}$ for an even more general solution (without the $w_{i}>0$ restriction).

Theorem 3 (Minimum $\sigma$-representation) Consider $X \sim$ $\left(\bar{X}, P_{X X}\right)^{n}, P_{X X}>0$. Then a MioR of $X$ is, for $v:=$ $\left[v_{1}, \ldots, v_{n}\right]^{T} \in \mathbb{R}^{n}, v_{i} \neq 0$, the set $\chi=\left\{\chi_{i}, w_{i}\right\}_{i=1}^{n+1}$ with

$$
\begin{aligned}
& w_{n+1}=\frac{1}{\sum_{i=1}^{n}\left(\left|w_{i}\right|+v_{i}^{2}\right)}, \quad|\bar{W}|^{-\frac{1}{2}} \bar{w}=\sqrt{w_{n+1}} v, \\
& \bar{E}:=\sqrt{P_{X X}}\left(\operatorname{sign}(\bar{W})+v v^{T}\right)^{-\frac{1}{2}}|\bar{W}|^{-\frac{1}{2}}, \\
& e:=-w_{n+1}^{-1} \bar{E} \bar{w}, \quad\left[\chi_{1}, \ldots, \chi_{n+1}\right]=[\bar{E}, e]+[\bar{X}]_{1 \times(n+1)} .
\end{aligned}
$$

Corollary 5 If $w_{i}>0$, then the normalized Mi $\sigma R$ of Theorem 3 becomes

$$
\begin{aligned}
& w_{n+1}=\frac{1}{1+\sum_{i=1}^{n} v_{i}^{2}}, \bar{w}=w_{n+1}\left[v_{1}^{2}, \ldots, v_{n}^{2}\right]^{T}, \\
& \bar{E}:=\sqrt{w_{n+1}^{-1} P_{X X}}\left(I+v v^{T}\right)^{-\frac{1}{2}} \operatorname{diag}(v)^{-1}, \\
& e:=-w_{n+1}^{-1} \bar{E} \bar{w}, \quad\left[\chi_{1}, \ldots, \chi_{n+1}\right]=[\bar{E}, e]+[\bar{X}]_{1 \times(n+1)} .
\end{aligned}
$$

If $w_{i}>0$ and $v=\rho C^{-1}[1]_{n \times 1}$, with $\rho$ and $C$ as in Tab I [6], then $\chi$ is the minimum sigma set of [12].

The Mi $\sigma \mathrm{R}$ from Theorem 3 is currently the only consistent $\sigma \mathrm{R}$ constituted by less than $2 n$ points, given that the set of [12] is a particular case of it and, to the best of the authors' knowledge, the other reduced sets do not fit Definition 1, i.e. the mean and/or covariance matrix of their prior distribution are not matched (see Section II-D). 


\section{UNSCENTED TRANSFORMATIONS}

\section{A. Unscented Transformation}

In this section, we reintroduce the definition of the Unscented Transformation (UT). In general terms, the UT consists of two sets of weighted points (the sigma points) that approximate a pdf of two rv's in the case where there is a functional dependence between them.

Henceforth, consider $Y:=f(X) \in \Phi^{n_{y}}, X \sim$ $\left(\bar{X}, M_{X}^{2}, \ldots, M_{X}^{l}\right)^{n}$. For the sets $\chi:=\left\{\chi_{i}, w_{i}^{(\mathbf{m})}, w_{i}^{\left(\mathbf{m}_{\lambda^{1} \ldots \lambda^{j}}^{j}\right)}\right.$ : $j \geq 2\}_{i=1}^{N} ; \gamma:=\left\{\gamma_{i}, w_{i}^{(\mathbf{m})}, w_{i}^{\left(\mathbf{m}_{\lambda^{1} \ldots \lambda j}^{j}\right)} \mid \gamma_{i}=f\left(\chi_{i}\right), j \geq\right.$ $2\}_{i=1}^{N}$; and vectors $\lambda^{\eta} \in\left\{\chi_{1}, \ldots, \chi_{N}, \gamma_{1}, \ldots, \gamma_{N}\right\}, \eta=1,2, \ldots$; the sample means are

$$
\mu_{\chi}:=\sum_{i=1}^{N} w_{i}^{\left(\mathbf{m}_{\chi}^{1}\right)} \chi_{i}, \quad \mu_{\gamma}:=\sum_{i=1}^{N} w_{i}^{\left(\mathbf{m}_{\gamma}^{1}\right)} \gamma_{i} .
$$

For even $j$, their sample central moments are

$$
\begin{aligned}
& \mathcal{M}_{\chi}^{j}:=\sum_{i=1}^{N} w_{i}^{\left(\mathbf{m}_{\chi_{1}, \ldots, \chi_{j}}^{j}\right)}\left[\left(\chi_{i}-\mu_{\chi}\right)\left(\chi_{i}^{T}-\mu_{\chi}^{T}\right)\right]^{\otimes \frac{j}{2}}, \\
& \left.\mathcal{M}_{\gamma}^{j}:=\sum_{i=1}^{N} w_{i}^{\left(\mathbf{m}_{\gamma_{1}}^{j}, \ldots, \gamma_{j}\right.}\right)\left[\left(\gamma_{i}-\mu_{\gamma}\right)\left(\gamma_{i}^{T}-\mu_{\gamma}^{T}\right)\right]^{\otimes \frac{j}{2}}, \\
& \left.\mathcal{M}_{\lambda^{1} \ldots \lambda^{j}}^{j}:=\sum_{i=1}^{N} w_{i}^{\left(\mathbf{m}_{\lambda 1}^{j} \ldots \lambda^{j}\right.}\right) \bigotimes_{q=1}^{j / 2}\left[\left(\lambda_{i}^{q}-\mu_{\lambda^{q}}\right)\left(\lambda_{i}^{q+1}-\mu_{\lambda^{(q+1)}}\right)^{T}\right] .
\end{aligned}
$$

For odd $j$, they are

$$
\begin{gathered}
\mathcal{M}_{\chi}^{j}:=\sum_{i=1}^{N} w_{i}^{\left(\mathbf{m}_{\chi_{1}, \ldots, \chi_{j}}^{j}\right)}\left[\left(\chi_{i}-\mu_{\chi}\right)\left(\chi_{i}^{T}-\mu_{\chi}^{T}\right)\right]^{\otimes \frac{j-1}{2}} \otimes\left(\chi_{i}-\mu_{\chi}\right), \\
\mathcal{M}_{\gamma}^{j}:=\sum_{i=1}^{N} w_{i}^{\left(\mathbf{m}_{\gamma_{1}, \ldots, \gamma_{j}}^{j}\right)}\left[\left(\gamma_{i}-\mu_{\gamma}\right)\left(\gamma_{i}^{T}-\mu_{\gamma}^{T}\right)\right]^{\otimes \frac{j-1}{2}} \otimes\left(\gamma_{i}-\mu_{\gamma}\right), \\
\mathcal{M}_{\lambda^{1} \ldots \lambda^{j}}^{j}:=\sum_{i=1}^{N} w_{i}^{\left(\mathbf{m}_{\lambda^{1} \ldots \lambda^{j}}^{j}\right)} \bigotimes_{q=1}^{(j-1) / 2} \\
{\left[\left(\lambda_{i}^{q}-\mu_{\lambda^{q}}\right)\left(\lambda_{i}^{q+1}-\mu_{\lambda^{(q+1)}}\right)^{T}\right] \otimes\left(\lambda_{i}^{j}-\mu_{\lambda^{j}}\right) .}
\end{gathered}
$$

Definition 2 (Unscented Transformation) Consider equations (19)-(25). If $\mu_{\chi}=\bar{X}$ and $\mathcal{M}_{\chi}^{j}=M_{X}^{j}, j=2, \ldots, l$, then the 1th order Unscented Transformation (IUT) is defined by

$$
\operatorname{lUT}\left(f, \bar{X}, M_{X}^{2}, \ldots, M_{X}^{l}\right)
$$

$$
:=\left[\mu_{\gamma}, \mathcal{M}_{\gamma}^{2}, \ldots, \mathcal{M}_{\gamma}^{l}, \mathcal{M}_{\lambda^{2} \lambda^{2}}^{2}, \ldots, \mathcal{M}_{\lambda^{1} \ldots \lambda^{l}}^{l}\right] .
$$

Remark 6 Every lthN $\sigma R$ is a set $\chi$ of a lUT.

This form of defining the $l \mathrm{UT}$ as a function mapping $(f, \bar{X}$, $\left.P_{X X}\right)$ to the transformed sample mean and CM's can also be used in Monte Carlo and quadrature methods. Moreover, one should notice that negative weights can lead to negative values of the sample moments.

A $l$ UT can be viewed as a function that maps $X$ and $Y$ to sets which approximate the $(\mathrm{X}, \mathrm{Y})$ joint pdf. For instance, a 2UT can be viewed as the approximation (this interpretation is inspired on [46])

$$
\left(\begin{array}{c}
X \\
Y
\end{array}\right) \approx\left(\begin{array}{c}
\tilde{X} \\
\tilde{Y}
\end{array}\right) \sim\left(\left(\begin{array}{c}
\mu_{\chi} \\
\mu_{\gamma}
\end{array}\right),\left(\begin{array}{cc}
\Sigma_{\chi \chi} & \Sigma_{\chi \gamma} \\
\Sigma_{\chi \gamma}^{T} & \Sigma_{\gamma \gamma}
\end{array}\right)\right)
$$

The next theorem states the approximation quality for the $Y$ 's. The notation $Y^{[c, l]}$ stands for the Taylor Series of $Y=$ $f(X)$ around $X=c$ truncated at the $l$ th order.

Theorem 4 If $\mathcal{M}_{\chi}^{j}=M_{X}^{j}, j=2, \ldots, l, \mu_{\chi}=\bar{X}$ and $f$ is lth differentiable, then:

1) $\mu_{\gamma}^{\left[\mu_{\chi}, l\right]}=\bar{Y}^{[\bar{X}, l]}$;

2) $\Sigma_{\gamma \gamma}^{\left[\mu_{\chi}, l / 2\right]}=P_{Y Y}^{[\bar{X}, l / 2]}$ if $l$ is even, $\Sigma_{\gamma \gamma}^{\left[\mu_{\chi},(l-1) / 2\right]}=P_{Y Y}^{\left[\bar{X}_{,},(l-1) / 2\right]}$ if $l$ is odd;

3) $\Sigma_{\chi \gamma}^{\left[\mu_{\chi}, l-1\right]}=P_{X Y}^{[\bar{X}, l-1]}$.

Proof: Suppose $\mu_{\chi}=\bar{X}$ and $\mathcal{M}_{\chi}^{p}=M_{X}^{p}, p=2, \ldots, l$. For the first assertion,

$$
\begin{aligned}
& \mu_{\gamma}^{\left[\mu_{\chi}, l\right]}=f\left(\mu_{\chi}\right)+\left.\frac{1}{2 !} \sum_{i_{1}, i_{2}=1}^{n}\left(\mathcal{M}_{\chi}^{2}\right)_{i_{1}, i_{2}} \frac{\partial^{2} f(x)}{\partial x^{\left(i_{1}\right)} \partial x^{\left(i_{2}\right)}}\right|_{x=\mu_{\chi}}+\ldots \\
& +\left.\frac{1}{l !} \sum_{i_{1}, \ldots, i_{l}=1}^{n}\left(\mathcal{M}_{\chi}^{l}\right)_{i_{1},\left(i_{2} * i_{3} * \ldots * i_{l}\right)} \frac{\partial^{l} f(x)}{\partial x^{\left(i_{1}\right)} \ldots \partial x^{\left(i_{l}\right)}}\right|_{x=\mu_{\chi}}=\bar{Y}^{[\bar{X}, l] .}
\end{aligned}
$$

For the second, $\Sigma_{\gamma \gamma}^{\left[l / 2, \mu_{\chi}\right]}=\Theta_{\Sigma_{\gamma \gamma}}^{2}+\ldots+\Theta_{\Sigma_{\gamma \gamma}}^{l / 2}$, where $\Theta_{\Sigma_{\gamma \gamma}}^{q}$ is given in (27), shown at the top of the next.

For the third assertion, $\Sigma_{\chi \gamma}^{\left[\mu_{\chi}, l-1\right]}=\Theta_{\Sigma_{\chi \gamma}}^{1}+\ldots+\Theta_{\Sigma_{\chi \gamma}}^{l-1}$, where

$$
\begin{aligned}
\Theta_{\Sigma_{\chi \gamma}}^{q}= & \frac{1}{q !}{ }_{i_{1}, \ldots, i_{q}=1}^{n}\left[\left(\mathcal{M}_{\chi}^{q+1}\right)_{1,\left(i_{1} * \ldots * i_{q}\right)}, \ldots,\left(\mathcal{M}_{\chi}^{q+1}\right)_{n,\left(i_{1} * \ldots * i_{q}\right)}\right]^{T} \\
& \left.\frac{\partial^{q} f(x)}{\partial x^{\left(i_{1}\right)} \ldots \partial x^{\left(i_{q}\right)}}\right|_{x=\mu_{\chi}} ^{T} .
\end{aligned}
$$

The remaining steps can be proven similarly.

Theorem 4 is the first to provide the estimation quality of the CCM, which is of the order $l-1\left(\Sigma_{\chi \gamma}^{\left[\mu_{\chi}, l-1\right]}=P_{X Y}^{[\bar{X}, l-1]}\right)$. The approximations of the posterior rv's are not guaranteed for any function $f$, but only for the $l$ th differentiable ones.

$$
\begin{aligned}
& \Theta_{\Sigma_{\gamma \gamma}}^{q}=\sum_{j=1}^{q-1}\left[\frac{1}{j ! q !} \sum_{i_{1}, \ldots i_{(q+j)}=1}^{n}\left(\left(\mathcal{M}_{\chi}^{q+j}\right)_{i_{1},\left(i_{2} * \ldots * i_{(q+j)}\right)}-\left(\mathcal{M}_{\chi}^{q}\right)_{i_{1},\left(i_{2} * \ldots * i_{q}\right)}\left(\mathcal{M}_{\chi}^{j}\right)_{i_{(l / 2+1)},\left(i_{(q+2)} * \ldots * i_{(q+j)}\right)}\right)\right) \\
& \left.\times\left(\left.\left.\frac{\partial^{q} f(x)}{\partial x^{\left(i_{1}\right)} \ldots \partial x^{\left(i_{q}\right)}}\right|_{x=\mu_{\chi}} \frac{\partial^{j} f(x)}{\left.\partial x^{\left(i^{(}(q+1)\right.}\right) \ldots \partial x^{\left(i_{(q+j)}\right)}}\right|_{x=\mu_{\chi}} ^{T}+\left.\left.\frac{\partial^{j} f(x)}{\left.\partial x^{\left(i^{(}(q+1)\right.}\right) \ldots \partial x\left(x^{(i q+j)}\right)}\right|_{x=\mu_{\chi}} \frac{\partial^{q} f(x)}{\partial x^{\left(i_{1}\right)} \ldots \partial x^{\left(i_{q}\right)}}\right|_{x=\mu_{\chi}} ^{T}\right)\right] \\
& +\frac{1}{q ! q !} \sum_{i_{2 q}=1}^{n}\left(\left(\mathcal{M}_{\chi}^{2 q}\right)_{i_{1},\left(i_{2} * \ldots * i_{2 q}\right)}-\left(\mathcal{M}_{\chi}^{q}\right)_{i_{1},\left(i_{2} * \ldots * i_{q}\right)}\left(\mathcal{M}_{\chi}^{q}\right)_{i_{(q+1)},\left(i_{(q+2)} * \ldots * i_{2 q}\right)}\right) \\
& \times\left.\left.\frac{\partial^{q} f(x)}{\partial x^{\left(i_{1}\right)} \ldots \partial x^{\left(i_{q}\right)}}\right|_{x=\mu_{\chi}} \frac{\partial^{q} f(x)}{\partial x^{\left(i_{(q+1)}\right)} \ldots \partial x^{\left(i_{2 q}\right)}}\right|_{x=\mu_{\chi}} ^{T} .
\end{aligned}
$$


For $l=2$, the transformed CM is approximated up to order 1 $\left(\Sigma_{\gamma \gamma}^{\left[\mu_{\chi}, 1\right]}=P_{Y Y}^{[\bar{X}, 1]}\right)$ (cf. Section II-C1).

According to Theorem 4, a sufficient condition for a second order approximation of the transformed $\mathrm{CM}$ is $l=4$, since, for even $l, \Sigma_{\gamma \gamma}^{\left[\mu_{\chi}, l / 2\right]}=P_{Y Y}^{[\bar{X}, l / 2]}$. In order to verify this, suppose $\mu_{\chi}=\bar{X}$ and $\mathcal{M}_{\chi}^{l}=M_{X}^{l}, i=2, \ldots, 4$. Then, from (27), $\Sigma_{\gamma \gamma}^{\left[\mu_{\chi}, 2\right]}=$ $\Theta_{\Sigma_{\gamma \gamma}}^{1}+\ldots+\Theta_{\Sigma_{\gamma \gamma}}^{2}=2 n=P_{Y Y}$. Moreover, consider $X \sim$ $N\left(0_{n \times 1}, I_{n}\right), Y:=f(X)=\left[x_{1}^{3}, \ldots, x_{n}^{3}\right]^{T}$ and suppose $\mu_{\chi}=\bar{X}$ and $\mathcal{M}_{\chi}^{l}=M_{X}^{l}, i=2, \ldots, 6$. Then, $\Sigma_{\gamma \gamma}^{\left[\mu_{\chi}, 3\right]}=\Theta_{\Sigma_{\gamma \gamma}}^{1}+\ldots+\Theta_{\Sigma_{\gamma \gamma}}^{3}=$ $15 n=P_{Y Y}$.

This result does not imply that the mean and CM estimates of a 2UT are equal to the ones obtained through linearization. We can point out two reasons. First, for a $2 \mathrm{UT}, \mu_{\gamma}^{\left[\mu_{\chi}, 2\right]}=\mu_{Y}^{[\bar{X}, 2]}$, whereas $\mu_{\chi}^{\left[\mu_{\chi}, 1\right]}=\mu_{Y}^{[\bar{X}, 1]}$ for linearization. Second, even though both linearization and a $2 \mathrm{UT}$ have $\Theta_{\Sigma_{\gamma \gamma}}^{1}=\Theta_{P_{Y Y}}^{1}$, it happens that, from (27), $\Theta_{\Sigma_{\gamma \gamma}}^{2}$ and $\Theta_{P_{Y Y}}^{2}$ are partially equal for a $2 \mathrm{UT}$, but not for linearization $\left(\Theta_{\Sigma_{\gamma \gamma}}^{2}=0\right)$.

\section{B. Scaled Unscented Transformation}

In this section, we redefine the Scaled Unscented Transformation (ScUT). This new definition is similar (not equal) to the AuxUT of [38] (Tab II [4:6]), since the SUT of [38] (Tab II [1:3]) cannot be applied to any previous sigma set (see Section II-E1). Furthermore, definitions similar to the previous SUT of [38] and to the UT of [35] (Tab I [7]) are presented at the end of this section as particular cases of the ScUT.

Unless otherwise specified, the term Scaled Unscented Transformation will henceforth refer to the following definition.

Definition 3 (Scaled Unscented Transformation) Consider, for $\alpha \in[0,1]$ and $\kappa \in(0,1]$, the function

$$
g(f, X, \beta, \alpha, \kappa)=\frac{f(\beta+\alpha(X-\beta))-f(\beta)}{\kappa}+f(\beta),
$$

and the sets $\chi:=\left\{\chi_{i}, w_{i}^{m}, w_{i}^{c}\right\}_{i=1}^{N}$ and $\gamma:=\left\{\gamma_{i}, w_{i}^{m}, w_{i}^{c} \mid \gamma_{i}=\right.$ $\left.g\left(f, \chi_{i}, \mu_{\chi}, \alpha, \alpha^{2}\right)\right\}_{i=1}^{N}$ and

$$
\begin{aligned}
& \Sigma_{\gamma \gamma}^{\alpha}:=\alpha^{2} \sum_{i=0}^{N} w_{i}^{c}\left(\gamma_{i}-\mu_{\gamma}\right)\left(\gamma_{i}-\mu_{\gamma}\right)^{T}, \\
& \Sigma_{\chi \gamma}^{\alpha}:=\alpha \sum_{i=0}^{N} w_{i}^{c}\left(\chi_{i}-\mu_{\chi}\right)\left(\gamma_{i}-\mu_{\gamma}\right)^{T},
\end{aligned}
$$

where $\Sigma_{\gamma \gamma}^{\alpha}$ is the scaled sample covariance matrix of $\gamma$ and $\Sigma_{\chi \gamma}^{\alpha}$ is the scaled sample cross covariance of $\chi$ and $\gamma$. If
$\mu_{\chi}=\bar{X}$ and $\Sigma_{\chi \chi}=P_{X X}$, then the Scaled Unscented Transformation (ScUT) is defined by

$$
\operatorname{ScUT}\left(f, \bar{X}, P_{X X}, \alpha\right):=\left[\mu_{\gamma}, \Sigma_{\gamma \gamma}^{\alpha}, \Sigma_{\chi \gamma}^{\alpha}\right] .
$$

\section{Remark 7 Every $2 t h N \sigma R$ is a set $\chi$ of a ScUT.}

Such a definition for the CCM of the ScUT cannot be found for the scaled UT's of the literature. Crossing covariances are not treated in the SUT of [38] or in the AuxUT of [38]. In the UT of [35], the CCM is defined differently and restricted only to the symmetric set defined there (see Section II-E3).

In Section II-E2 and II-E3, it was shown that $\alpha$ modifies the second order terms of both $\Sigma_{\gamma \gamma}^{\alpha}$ and $\Sigma_{\chi \gamma}$. In order to check the influence of $\alpha$ in the covariances of the ScUT, define $\Theta_{\Sigma_{\gamma \gamma}^{\alpha}}^{l}$ and $\Theta_{\Sigma_{\gamma \gamma}^{\alpha}}^{l}$ as the $l$ th term of the Taylor Series of $\Sigma_{\gamma \gamma}^{\alpha}$ and $\Sigma_{\chi \gamma}^{\alpha}$, respectively, and consider the equations at the bottom of the page. The ScUT scales the terms of order 3 and higher for $\mu_{\gamma}$ and of order 2 and higher for $\Sigma_{\gamma \gamma}^{\alpha}$ and $\Sigma_{\chi \gamma}^{\alpha}$. However, if $\chi$ is symmetric, then $\mathcal{M}_{\chi}^{3}=[0]_{n \times 2 n} \Rightarrow \Theta_{\Sigma_{\chi \gamma}^{\alpha}}^{3}=[0]_{n \times 1}$ and $\alpha$ does not modify the second order of $\Sigma_{\chi \gamma}^{\alpha}$ (cf. Section II-E3). The next theorem gives the estimation quality of the ScUT.

Theorem 5 If $\Sigma_{\chi \chi}=P_{X X}, \mu_{\chi}=\bar{X}$ and $f$ is 2nd-order differentiable, then $\mu_{\gamma}^{\left[\mu_{\chi}, 2\right]}=\bar{Y}^{[\bar{X}, 2]}, \Sigma_{\gamma \gamma}^{\alpha,\left[\mu_{\chi}, 1\right]}=P_{Y Y}^{[\bar{X}, 1]}$ and $\Sigma_{\chi \gamma}^{\alpha,\left[\mu_{\chi}, 1\right]}=P_{X Y}^{[\bar{X}, 1]}$. Furthermore, if $X$ and $\chi$ are symmetric, then $\Sigma_{\chi \gamma}^{\alpha,\left[\mu_{\chi}, 2\right]}=P_{X Y}^{[\bar{X}, 2]}$.

Proof: Suppose $\mu_{\chi}=\bar{X}, \Sigma_{\chi \chi}=P_{X X}$. For the first assertion,

$\mu_{\gamma}^{\left[\mu_{\chi}, 2\right]}=f\left(\mu_{\chi}\right)+\left.\frac{1}{2 !} \sum_{i_{1}, i_{2}=1}^{n}\left(\mathcal{M}_{\chi}^{2}\right)_{i_{1}, i_{2}} \frac{\partial^{2} f(x)}{\partial x^{\left(i_{1}\right)} \partial x^{\left(i_{2}\right)}}\right|_{x=\mu_{\chi}}=\bar{Y}^{[\bar{X}, 2]}$,

which proves the first assertion. For the second assertion,

$$
\Sigma_{\gamma \gamma}^{\alpha,\left[\mu_{\chi}, 1\right]}=\left.\left.\sum_{i, j=1}^{n}\left(\Sigma_{\chi \chi}\right)_{i, j} \frac{\partial f(x)}{\partial x^{(i)}}\right|_{x=\mu_{\chi}} \frac{\partial f(x)}{\partial x^{(j)}}\right|_{x=\mu_{\chi}} ^{T}=P_{Y Y}^{\left[\mu_{\chi}, 1\right]} .
$$

For the third assertion,

$$
\Sigma_{\chi \gamma}^{\alpha\left[\mu_{\chi}, 1\right]}=\left.\sum_{i=1}^{n}\left[\left(\Sigma_{\chi \chi}\right)_{1, i}, \ldots,\left(\Sigma_{\chi \chi}\right)_{n, i}\right]^{T} \frac{\partial f(x)}{\partial x^{(i)}}\right|_{x=\mu_{\chi}} ^{T}=P_{X Y}^{[\bar{X}, 1]} .
$$

For the last assertion, note that $X$ symmetric implies $M_{\chi}^{3}=$ $[0]_{n \times 2 n} \Rightarrow \Theta_{P X Y}^{3}=[0]_{n \times 1}$ and $\chi$ symmetric implies $\mathcal{M}_{\chi}^{3}=$ $[0]_{n \times 2 n} \Rightarrow \Theta_{\Sigma_{\chi \gamma}^{\alpha}}^{3}=[0]_{n \times 1}$.

$$
\begin{aligned}
& \mu_{\gamma}=f\left(\mu_{\chi}\right)+\left.\sum_{i_{1}, i_{2}=1}^{n}\left(\mathcal{M}_{\chi}^{2}\right)_{i_{1}, i_{2}} \frac{\partial^{2} f(x)}{\partial x^{\left(i_{1}\right)} \partial x^{\left(i_{2}\right)}}\right|_{x=\mu_{\chi}}+\ldots+\left.\alpha^{l-2} \sum_{i_{1}, \ldots, i_{l}=1}^{n}\left(\mathcal{M}_{\chi}^{l}\right)_{i_{1}, i_{2} * \ldots * i_{l}} \frac{\partial^{l} f(x)}{\partial x^{\left(i_{1}\right)} \ldots \partial x^{\left(i_{l}\right)}}\right|_{x=\mu_{\chi}}+\ldots \\
& \Theta_{\Sigma_{\gamma \gamma}^{\alpha}}^{l}=\sum_{j=1}^{l-1}\left[\frac{\alpha^{j+l-2}}{j ! l !} \sum_{i_{1}, \ldots i_{l+j}=1}^{n}\left(\left(\mathcal{M}_{\chi}^{l+j}\right)_{i_{1},\left(i_{2} * \ldots * i_{(l+j)}\right)}-\left(\mathcal{M}_{\chi}^{l}\right)_{i_{1},\left(i_{2} * \ldots * i_{l}\right)}\left(\mathcal{M}_{\chi}^{j}\right)_{i_{(l+1)},\left(i_{(l+2)} * \ldots * i_{(l+j)}\right)}\right)\right. \\
& \left.\left(\left.\left.\left.\frac{\partial^{l} f(x)}{\partial x^{\left(i_{1}\right)} \ldots \partial x^{\left(i_{l}\right)}}\right|_{x=\mu_{\chi}} \frac{\partial^{j} f(x)}{\partial x^{\left(i_{l+1}\right)} \ldots \partial x}\right|^{\left(i_{l+j}\right)}\right|_{x=\mu_{\chi}} ^{T}+\left.\left.\frac{\partial^{j} f(x)}{\partial x^{\left(i_{l+1}\right)} \ldots \partial x{ }^{\left(i_{l+j}\right)}}\right|_{x=\mu_{\chi}} \frac{\partial^{l} f(x)}{\partial x^{\left(i_{1}\right)} \ldots \partial x^{\left(i_{l}\right)}}\right|_{x=\mu_{\chi}} ^{T}\right)\right] \\
& +\frac{\alpha^{2 l-2}}{l ! l !} \sum_{i_{1}, \ldots, i_{2 l}=1}^{n}\left(\left(\mathcal{M}_{\chi}^{2 l}\right)_{i_{1},\left(i_{2} * \ldots * i_{2 l}\right)}-\left(\mathcal{M}_{\chi}^{l}\right)_{i_{1},\left(i_{2} * \ldots * i_{l}\right)}\left(\mathcal{M}_{\chi}^{l}\right)_{i_{(l+1)},\left(i_{(l+2)} * \ldots * i_{2 l}\right)}\right) \\
& \left.\left.\frac{\partial^{l} f(x)}{\partial x^{\left(i_{1}\right)} \ldots \partial x^{\left(i_{l}\right)}}\right|_{x=\mu_{\chi}} \frac{\partial^{l} f(x)}{\left.\partial x^{\left({ }^{(}(l+1)\right.}\right) \ldots \partial x^{\left(i_{2 l}\right)}}\right|_{x=\mu_{\chi}} ^{T} \\
& \Theta_{\Sigma_{\chi \gamma}^{\alpha}}^{l}=\left.\alpha^{l-1} \frac{1}{l !} \sum_{i_{1}, \ldots, i_{l}=1}^{n}\left[\left(\mathcal{M}_{\chi}^{l+1}\right)_{1,\left(i_{1} * \ldots * i_{l}\right)}, \ldots,\left(\mathcal{M}_{\chi}^{l+1}\right)_{n,\left(i_{1} * \ldots * i_{l}\right)}\right]^{T} \frac{\partial^{l} f(x)}{\partial x^{\left(i_{1}\right)} \ldots \partial x^{\left(i_{l}\right)}}\right|_{x=\mu_{\chi}} ^{T}
\end{aligned}
$$


Similar to the 2UT, the CM of the transformed rv is estimated only up to first order. Theorem 5 gives, for the first time, the estimation quality of the sample CCM. The next corollary states a new result.

Corollary 6 A ScUT with sets $\left\{\chi_{i}, w_{i}^{m}, w_{i}^{c}\right\}_{i=1}^{N} \quad$ and $\left\{\gamma_{i}, w_{i}^{m}, w_{i}^{c} \mid \gamma_{i}=g\left(f, \chi_{i}, \mu_{\chi}, \alpha, \alpha^{2}\right)\right\}_{i=1}^{N}$ is a 2UT with sets $\left\{\chi_{i}, w_{i}^{m}, w_{i}^{c}\right\}_{i=1}^{N} \quad$ and $\left\{\gamma_{i}, w_{i}^{m}, w_{i}^{\alpha, c}, w_{i}^{\alpha, c c} \mid \gamma_{i}=\right.$ $\left.g\left(f, \chi_{i}, \mu_{\chi}, \alpha, \alpha^{2}\right)\right\}_{i=1}^{N}$ where $w_{i}^{\alpha, c}=\alpha^{2} w_{i}^{c}$ and $w_{i}^{\alpha, c c}=\alpha w_{i}^{c}$ are the weights to calculate the sample CM and CCM, respectively.

Because of the way these transformations were defined, every ScUT is a $2 \mathrm{UT}$ and, therefore, every result obtained for the $2 \mathrm{UT}$ can also be applied to the ScUT. We proceed by redefining the SUT of [38] and the UT of [35].

Definition 4 Let $\chi:=\left\{\chi_{i}, w_{i}\right\}_{i=1}^{N}$ be a normalized $\sigma R$ of $X$ with $\chi_{N}=\bar{X}$, and consider the sets $\chi^{\prime}:=\left\{\chi_{i}^{\prime}, w_{i}^{\prime} \mid \chi_{i}^{\prime}=\chi_{N}+\right.$ $\left.\alpha\left(\chi_{i}-\chi_{N}\right)\right\}_{i=1}^{N}$ and $\gamma^{\prime}:=\left\{\gamma_{i}^{\prime}, w_{i}^{\prime} \mid \gamma_{i}^{\prime}=f\left(\chi_{i}^{\prime}\right)\right\}_{i=1}^{N}$ where, for $\alpha \in(0,1], w_{N}^{\prime}:=\alpha^{-2} w_{N}+1-\alpha^{-2}, w_{i}^{\prime}=\alpha^{-2} w_{i}, i=$ $1, \ldots, N-1$. Define the modified sample $C M$ and the sample CCM of $\gamma^{\prime}$, respectively, as

$$
\Sigma_{\gamma^{\prime} \gamma^{\prime}}^{\alpha \alpha}:=\sum_{i=1}^{N} w_{i}^{\prime}\left(\gamma_{i}^{\prime}-\mu_{\gamma^{\prime}}\right)(\diamond)^{T}+\left(1-\alpha^{2}\right)\left(\gamma_{N}^{\prime}-\mu_{\gamma^{\prime}}\right)(\diamond)^{T}
$$

and $\Sigma_{\chi^{\prime} \gamma^{\prime}}:=\sum_{i=1}^{N} w_{i}^{\prime}\left(\chi_{i}^{\prime}-\mu_{\chi^{\prime}}\right)\left(\gamma_{i}^{\prime}-\mu_{\gamma^{\prime}}\right)^{T}$. Then the Simplex Scaled Unscented Transformation (SiScUT) is defined by

$$
\operatorname{SiScUT}\left(f, \bar{X}, P_{X X}, \alpha\right):=\left[\mu_{\gamma^{\prime}}, \Sigma_{\gamma^{\prime} \gamma^{\prime}}^{\alpha \alpha}, \Sigma_{\chi^{\prime} \gamma^{\prime}}\right] .
$$

Definition 5 Let $\chi:=\left\{\chi_{i}, w_{i}\right\}_{i=1}^{2 n+1}$ with $\chi_{2 n+1}=\bar{X}$ and $w_{2 n+1}=\lambda /(n+\lambda)$ for $\alpha \in(0,1], \kappa \in \mathbb{R} \backslash\{-n\}$ and $\lambda=\alpha^{2}(n+\kappa)+n \neq 0$ be a normalized HoMiSy $\sigma R$ of $X$ and consider the set $\tilde{\gamma}:=\left\{\tilde{\gamma}_{i}, \tilde{w}_{i}^{m}, \tilde{w}_{i}^{c}, \tilde{w}_{i}^{c c} \mid \tilde{\gamma}_{i}=f\left(\chi_{i}\right)\right\}_{i=1}^{2 n+1}$ where $\tilde{w}_{2 n+1}^{m}=w_{2 n+1}, \tilde{w}_{2 n+1}^{c}=w_{2 n+1}+\left(1-\alpha^{2}\right)$, $\tilde{w}_{2 n+1}^{c c}=w_{2 n+1}+(1-\alpha), \tilde{w}_{i}^{m}=\tilde{w}_{i}^{c}=\tilde{w}_{i}^{c c}=w_{i}$, $i=1, \ldots, 2 n$ and $\tilde{w}_{i}^{c c}$ is the weight for calculating the sample CCM. Then the Symmetric Intrinsically Scaled Unscented Transformation (SyInScUT) is defined by

$$
\operatorname{SyInScUT}\left(f, \bar{X}, P_{X X}, \alpha\right):=\left[\mu_{\tilde{\gamma}}, \Sigma_{\tilde{\gamma} \tilde{\gamma}}, \Sigma_{\chi \tilde{\gamma}}\right] .
$$

Corollary 7 Every SiScUT and every SyInScUT are ScUTS.

Proof: Let $\chi$ and $\gamma$ be the sets of a ScUT (Definition 3) with $w_{i}^{c}=w_{i}^{m}=w_{i}$. To prove the first part, consider Definition 4. First, from (19), $\mu_{\gamma}=\left(1-w_{N}\right)\left(1-\alpha^{-2}\right) \gamma_{N}^{\prime}+$ $\sum_{i=1}^{N-1} \alpha^{-2} w_{i} \gamma_{i}^{\prime}+w_{N} \gamma_{N}^{\prime}=\mu_{\gamma^{\prime}}$. Second, from (28), $\Sigma_{\gamma \gamma}^{\alpha}=$ $\sum_{i=1}^{N-1} w_{i}^{\prime}\left(\gamma_{i}^{\prime}-\mu_{\gamma^{\prime}}\right)(\diamond)^{T}+\alpha^{-2}\left(1-\alpha^{2}\right)\left(\gamma_{N}^{\prime}-\mu_{\gamma^{\prime}}\right)(\diamond$ )$^{T}-\alpha^{-2}\left(\alpha^{2}-1\right)^{2}\left(\mu_{\gamma^{\prime}}-\gamma_{N}^{\prime}\right)(\diamond)^{T}=\Sigma_{\gamma^{\prime} \gamma^{\prime}}^{\alpha \alpha}$. Third, from (28), $\Sigma_{\chi \gamma}^{\alpha}=\sum_{i=1}^{N} w_{i}^{\prime}\left(\chi_{i}^{\prime}-\mu_{\chi^{\prime}}\right)\left(\gamma_{i}^{\prime}-\mu_{\gamma^{\prime}}\right)^{T}=\Sigma_{\chi^{\prime} \gamma^{\prime}}$. The remaining steps of the first part are trivial.

To prove the second part, consider Definition 5 and define the set $\tilde{\varsigma}:=\left\{\tilde{\varsigma}_{i}, \tilde{w}_{i} \mid \tilde{\varsigma}_{i}=\gamma_{i}\right\}_{i=1}^{2 n+1}$, where $\tilde{w}_{2 n+1}:=$ $\alpha^{-2} w_{2 n+1}+1-\alpha^{-2}, \tilde{w}_{i}:=\alpha^{-2} w_{i}, i=1, \ldots, 2 n$, and note that, from Definition 4 , the function $\Gamma\left(f, \bar{X}, P_{X X}, \alpha\right):=$ $\left[\mu_{\tilde{\varsigma}}, \Sigma_{\tilde{\varsigma} \tilde{\varsigma}}^{\alpha}, \Sigma_{\chi \tilde{\varsigma}}\right]$ is a SiScUT. Then it can easily be proven that $\mu_{\gamma}=\mu_{\tilde{\varsigma}}, \Sigma_{\gamma \gamma}=\Sigma_{\tilde{\varsigma} \tilde{\varsigma}}^{\alpha}$ and $\Sigma_{\chi \gamma}=\Sigma_{\chi \tilde{\varsigma}}$.

The SUT of [38] is incorporated in the SiScUT (Definition 4) whith the difference that now it states the restriction of having a point located in the mean (cf. Section II-E1) and defines the sample CCM (cf. Section II-E2). Besides, with Corollary 7, the SiScUT follows naturally as a particular case of the ScUT and, therefore, we also have the estimation quality of $P_{Y Y}$ and $P_{X Y}$ and the influence of $\alpha$ on the estimate of $P_{X Y}$ (see Section II-E). Definition 5 provides similar results for the UT of [35] which we now define as SyInScUT. Summing up, we provide unified and consistent new definitions for all the scaled transformations.

\section{Square-root Unscented Transformation}

In this Section, we state the results for the Square-Root Unscented Transformation (SRUT). As Section II-F3 pointed out, Definition 6 should be the first definition for a SRUT.

The key idea of a SRUT is to transform the square-root matrix of the previous CM directly (without squaring) into the square-root matrix of the posterior CM. For instance, one way of doing it for $\Sigma_{\gamma \gamma}^{Q}:=\Sigma_{\gamma \gamma}+\sqrt{Q} \sqrt{Q}^{T}$ is by the function

$$
c u\left(S_{\gamma}^{+}, S_{\gamma}^{-}, \sqrt{Q}\right):=\sqrt{\Sigma_{\gamma \gamma}^{Q}}
$$

where, for a set $\gamma=\left\{\gamma_{i}, w_{i}^{m}, w_{i}^{c}\right\}_{i=1}^{N}$, we define

$$
\begin{aligned}
& \gamma_{+}:=\left\{\gamma_{j,+}, w_{j,+}^{m}, w_{j,+}^{c}\right\}_{j=1}^{N_{+}}=\left\{\gamma_{i}, w_{i}^{m}, w_{i}^{c} \mid w_{i}^{c} \geq 0\right\}_{i=1}^{N}, \\
& \gamma_{-}:=\left\{\gamma_{j,-}, w_{j,-}^{m}, w_{j,-}^{c}\right\}_{j=1}^{N_{-}}=\left\{\gamma_{i}, w_{i}^{m}, w_{i}^{c} \mid w_{i}^{c}<0\right\}_{i=1}^{N}, \\
& S_{\gamma}^{+}:=\left[\sqrt{w_{1,+}^{c}}\left(\gamma_{1,+}-\mu_{\gamma}\right), \ldots, \sqrt{w_{N_{+},+}^{c}}\left(\gamma_{N_{+},+}-\mu_{\gamma}\right)\right], \\
& S_{\gamma}^{-}:=\left[\sqrt{\left\|w_{1,-}^{c}\right\|}\left(\gamma_{1,-}-\mu_{\gamma}\right), \ldots, \sqrt{\left\|w_{N_{-},-}^{c}\right\|}\left(\gamma_{N_{-},-}-\mu_{\gamma}\right)\right],
\end{aligned}
$$

and $\sqrt{\Sigma_{\gamma \gamma}^{Q}}$ is calculated by the following algorithm:

1. $\Phi=\operatorname{tria}\left\{\left[S_{\gamma}^{+}, \sqrt{Q}\right]\right\}$;

2. If $N_{-}>0, \sqrt{\Sigma_{\gamma \gamma}^{Q}}=\operatorname{cdown}\left\{\Phi, S_{\gamma}^{-}\right\}$; else, $\sqrt{\Sigma_{\gamma \gamma}^{Q}}=\Phi$.

In this way, $\sqrt{\Sigma_{\gamma \gamma}^{Q}}$ can be obtained by first updating the Cholesky factor, and then downdating it. The former operation can be done by means of triangularization (e.g. the QR decomposition) $S=\operatorname{tria}\{A\}, A \in \mathbb{R}^{n \times n}$, where $S$ is lower triangular (see [6], [48]). The latter can be achieved through $S=\operatorname{cdown}\{A, B\}, B \in \mathbb{R}^{n \times n_{y}}$, representing the Cholesky downdating of $A$ by $B$ (it is the same as doing cholupdate $\left\{A, B_{*, i},-1\right\}$ [43] for $\left.i=1, \ldots, n_{y}\right)$. Note, nevertheless, that downdating the Cholesky factor might lead to ill-conditioned matrices [47] (see Section II-F1). Therefore, this procedure, which is only necessary when the $\sigma \mathrm{R}$ contains negative weights, should be avoided whenever possible. We can, therefore, define the SRUT. For now on, consider the rv $X$ charactezised by the mean $\bar{X}$ and square-root of the CM $\sqrt{P_{X X}}$.

\section{Definition 6 (Square-Root Unscented Transformation)}

Consider the sets $\chi=\left\{\chi_{i}, w_{i}\right\}$ and $\gamma=\left\{\gamma_{i}, w_{i} \mid \gamma_{i}=f\left(\chi_{i}\right)\right\}$ with $\mu_{\chi}=\bar{X}$ and $\Sigma_{\chi \chi}=\sqrt{P_{X X}} \sqrt{P_{X X}}$. Given a matrix $\sqrt{Q}$, the Square-Root Unscented Transformation (SRUT) is defined by

$\operatorname{SRUT}\left(f, \bar{X}, \sqrt{P_{X X}}, \sqrt{Q}\right):=\left[\mu_{\gamma}, \sqrt{\Sigma_{\gamma \gamma}^{Q}}, S_{\chi}^{+}, S_{\chi}^{-}, S_{\gamma}^{+}, S_{\gamma}^{-}, \Sigma_{\chi \gamma}\right]$. 
Next, we introduce the Scaled SRUT and some results concerning this Transformation. This definition is necessary for the Scaled SRUKF's (Section V-B), the first one in the literature.

Definition 7 (Scaled Square-Root UT) Consider the sets $\chi$ and $\gamma$ as in Definition 3 with $\mu_{\chi}=\bar{X}$ and $\Sigma_{\chi \chi}=$ $\sqrt{P_{X X}}{\sqrt{P_{X X}}}^{T}$. Given a matrix $\sqrt{Q}$, define $\Sigma_{\gamma \gamma}^{\alpha Q}:=\Sigma_{\gamma \gamma}^{\alpha}+$ $\sqrt{Q} \sqrt{Q}^{T}$. Then the Scaled Square-Root Unscented Transformation (ScSRUT) is defined by

$\operatorname{ScSRUT}\left(f, \bar{X}, \sqrt{P_{X X}}, \sqrt{Q}, \alpha\right):=\left[\mu_{\gamma}, \sqrt{\Sigma_{\gamma \gamma}^{\alpha Q}}, S_{\chi}^{+}, S_{\chi}^{-}, S_{\gamma}^{+}, S_{\gamma}^{-}, \Sigma_{\chi \gamma}^{\alpha}\right]$.

Corollary 8 A ScSRUT with sets $\chi=\left\{\chi_{i}, w_{i}^{m}, w_{i}^{c}\right\}_{i=1}^{N}$ and $\left\{\gamma_{i}, w_{i}^{m}, w_{i}^{c} \mid \gamma_{i}=g\left(f, \chi_{i}, \mu_{\chi}, \alpha, \alpha^{2}\right)\right\}_{i=1}^{N}$ is a SRUT with the sets $\chi$ and $\left\{\gamma_{i}, w_{i}^{m}, \alpha^{2} w_{i}^{c}, \alpha w_{i}^{c}\right\}$.

Remark 8 Every 2 thN $\sigma R$ is a set $\chi$ of a SRUT.

Finally, we state new ScSRUT results similar to the ones in Section IV-B for the particular scaled transformations.

Definition 8 Consider the sets $\chi^{\prime}$ and $\gamma^{\prime}$ as in Definition 4 with $\mu_{\chi^{\prime}}=\bar{X}$ and $\Sigma_{\chi^{\prime} \chi^{\prime}}=\sqrt{P_{X X}}{\sqrt{P_{X X} X}}^{T}$. Given a matrix $\sqrt{Q}$, define $\Sigma_{\gamma^{\prime} \gamma^{\prime}}^{\alpha \alpha Q}:=\Sigma_{\gamma^{\prime} \gamma^{\prime}}^{\alpha \alpha}+\sqrt{Q} \sqrt{Q}^{T}$. Then the Simplex Scaled Square-Root Unscented Transformation (SiScSRUT) is defined by

$$
\begin{aligned}
\operatorname{SiScSRUT} & \left(f, \bar{X}, \sqrt{P_{X X}}, \sqrt{Q}, \alpha\right):= \\
& {\left[\mu_{\gamma^{\prime}}, \sqrt{\Sigma_{\gamma^{\prime} \gamma^{\prime}}^{\alpha \alpha Q}}, S_{\chi^{\prime}}^{+}, S_{\chi^{\prime}}^{-}, S_{\gamma^{\prime}}^{+}, S_{\gamma^{\prime}}^{-}, \Sigma_{\chi^{\prime} \gamma^{\prime}}\right] . }
\end{aligned}
$$

Definition 9 Consider the sets $\tilde{\chi}$ and $\tilde{\gamma}$ as in Definition 5 with $\mu_{\tilde{\chi}}=\bar{X}$ and $\Sigma_{\tilde{\chi} \tilde{\chi}}=\sqrt{P_{X X}} \sqrt{P_{X X}}{ }^{T}$. Given a matrix $\sqrt{Q}$, define $\Sigma_{\tilde{\gamma} \tilde{\gamma}}^{Q}:=\Sigma_{\tilde{\gamma} \tilde{\gamma}}+\sqrt{Q} \sqrt{Q}^{T}$. Then the Symmetric Intrinsically Scaled Square-Root Unscented Transformation (SyInScSRUT) is defined by

$$
\begin{aligned}
\operatorname{SyInSCSRUT}( & \left.f, \bar{X}, \sqrt{P_{X X}}, \sqrt{Q}, \alpha\right):= \\
& {\left[\mu_{\tilde{\gamma}}, \sqrt{\Sigma_{\tilde{\gamma} \tilde{\gamma}}^{Q}}, S_{\tilde{\chi}}^{+}, S_{\tilde{\chi}}^{-}, S_{\tilde{\gamma}}^{+}, S_{\tilde{\gamma}}^{-}, \Sigma_{\tilde{\chi} \tilde{\gamma}}\right] . }
\end{aligned}
$$

Corollary 9 Every SiScSRUT is an ScSRUT and every SyInScSRUT is an SCSRUT.

\section{UnSCENTED KaLman Filters}

In this section, we present discrete-time Unscented Kalman Filters. These are recursive applications of the UT (Section IV) to the discrete-time stochastic filtering problem (see Section II) in a KF framework.

\section{A. Unscented Kalman Filter}

For the sake of clarity of presentation, we only explicitly describe the Unscented Kalman Filter for the system in additive form (1). The general filter for (2) can be similarly obtained by considering the augmented functions $f^{a}: \mathbb{R}^{n_{a}} \rightarrow \mathbb{R}^{n_{x}}$ and $h^{a}: \mathbb{R}^{n_{a}} \rightarrow \mathbb{R}^{n_{y}}$ such that, for $x_{k-1 \mid k-1}^{a}:=\left[\hat{x}_{k-1 \mid k-1}^{T}, q_{k}^{T}, r_{k}^{T}\right]^{T}$,

$$
\begin{aligned}
& f^{a}\left(x^{a}, k\right)=f\left(x_{\left(1: n_{x}\right), 1}^{a}, x_{\left(n_{x}+1: n_{x}+n_{q}\right), 1}^{a}, k\right), \\
& h^{a}\left(x^{a}, k\right)=h\left(x_{\left(1: n_{x}\right), 1}^{a}, x_{\left(n_{x}+n_{q}+1: n_{a}\right), 1}^{a}, k\right) .
\end{aligned}
$$

Additive UKF (AdUKF): Consider (1),

1) $\hat{x}_{k-1 \mid k-1}$ and $\hat{P}_{x x}^{k-1 \mid k-1}$ are given.

2) Obtain the predicted statistics of the state:

$$
\begin{aligned}
{\left[\hat{x}_{k \mid k-1}, \hat{P}_{x x, *}^{k \mid k-1}\right] } & =U T_{1}\left(f, \hat{x}_{k-1 \mid k-1}, \hat{P}_{x x}^{k-1 \mid k-1}\right), \\
\hat{P}_{x x}^{k \mid k-1} & =\hat{P}_{x x, *}^{k \mid k-1}+Q_{k} .
\end{aligned}
$$

3) Obtain the predicted statistics of the measurement:

$$
\begin{aligned}
{\left[\hat{y}_{k \mid k-1}, \hat{P}_{y y, *}^{k \mid k-1}, \hat{P}_{x y}^{k \mid k-1}\right] } & =U T_{2}\left(h, \hat{x}_{k \mid k-1}, \hat{P}_{x x}^{k \mid k-1}\right), \\
\hat{P}_{y y}^{k \mid k-1} & =\hat{P}_{y y, *}^{k \mid k-1}+R_{k} .
\end{aligned}
$$

4) Obtain the corrected statistics of the state by (7).

Given that we only consider the second order UT in this subsection, we use the notation $U T$ to refer to the $2 U T$ (higher order UKFs are considered in Section V-E). The notations $U T_{1}$ and $U T_{2}$ (and, analogously, $S R U T_{1}$ and $S R U T_{2}$ for the square-root filters in the next subsection) indicate that the transformations in the prediction and correction steps do not need to be the same. In fact, the number of sigma points can be different, and we could even use the $S c U T$. The output of $U T_{1}\left(S R U T_{1}\right)$ has only two terms meaning that only the first two elements of the output of Definition 2 (Definition 6) are needed in the algorithm. One should also consider that if $f$ (or $h$ ) is linear, then $U T_{1}\left(U T_{2}\right)$ can be substituted by the linear Kalman Filter prediction (correction) equations.

The set $\chi^{k \mid k-1}=\left\{\chi_{i}^{k \mid k-1}, w_{i}\right\}$ is regenerated in step 3), since it is the previous $\sigma$-representation of $U T_{2}$. One can consider not doing so by propagating $\chi^{k \mid k-1}$ already obtained in step 2) (the posterior set of $U T_{1}$ ), but, as [32] points out, $\chi^{k \mid k-1}$ would not carry information about the process noise [cf. (3) and (4)].

\section{B. Square-Root Unscented Kalman Filter}

We now present the Square-Root Unscented Kalman Filter (SRUKF). The main difference between this filter and other types of UKF is the fact that the SRUKF propagate the squareroot matrix of the CM's directly, which is computationally more stable than squaring the propagated CM [48].

As Section II-F1 pointed out, the SRUKF's in the literature present three steps in which Cholesky factors are downdated: in the calculations of the square-root matrices of the CM for the predicted state; in the $\mathrm{CM}$ for the innovation; and in the $\mathrm{CM}$ for the corrected state. While, in the first two cases, downdating is only performed when negative weights exist, the last one is always performed. Due to the fact that downdating steps can be computationally unstable (see Section II-F1), we derive an alternative form - which is an extension of the results of [48] and [6] - that uses the downdating procedure only for the negative weight components.

Define $S_{\gamma}^{+}, S_{\gamma}^{-}, S_{\chi}^{+}, S_{\chi}^{-}$as in Section IV-C and note that

$$
\hat{P}_{x x}^{k \mid k-1}=S_{\chi}^{+} S_{\chi}^{+T}-S_{\chi}^{-} S_{\chi}^{-T}, \hat{P}_{y y}^{k \mid k-1}=S_{\gamma}^{+} S_{\gamma}^{+T}-S_{\gamma}^{-} S_{\gamma}^{-T}
$$

and $\hat{P}_{x x}^{k \mid k-1}=S_{\chi}^{+} S_{\gamma}^{+T}-S_{\chi}^{-} S_{\gamma}^{-T}+R_{k}$. Therefore, $\hat{P}_{x x}^{k \mid k}=\left[S_{\chi}^{+}-G_{k} S_{\gamma}^{+}, G_{k} R\right][\diamond]^{T}-\left[S_{\chi}^{-}-G_{k} S_{\gamma}^{-}, G_{k} R\right][\diamond]^{T}$, 
which shows that $\hat{P}_{x x}^{k \mid k}$ can be obtained through updating and downdating. The latter is only performed for the negative weight cases.

The SRUKF is presented below. It is more general than the algorithms currently in the literature, since these are restricted to the case where only the central weight, $w_{0}$, can be negative, whereas our SRUKF does not restrict the quantity of negative weights.

Additive SRUKF (AdSRUKF): Consider (1),

1) $\hat{x}_{k-1 \mid k-1}$ and $\sqrt{\hat{P}_{x x}^{k-1 \mid k-1}}$ are given.

2) Obtain the predicted statistics of the state:

$\left[\hat{x}_{k \mid k-1}, \sqrt{\hat{P}_{x x}^{k \mid k-1}}\right]=\operatorname{SRUT}_{1}\left(f, \hat{x}_{k-1 \mid k-1}, \sqrt{\hat{P}_{x x}^{k-1 \mid k-1}}, \sqrt{Q_{k}}\right)$.

3) Obtain the predicted statistics of the measurement:

$$
\begin{aligned}
{\left[\hat{y}_{k \mid k-1}, \sqrt{\hat{P}_{y y}^{k \mid k-1}}, S_{\chi}^{+}, S_{\chi}^{-}, S_{\gamma}^{+}, S_{\gamma}^{-}, \hat{P}_{x y}^{k \mid k-1}\right]=} \\
\operatorname{SRUT}_{2}\left(h, \hat{x}_{k \mid k-1}, \sqrt{\hat{P}_{x x}^{k \mid k-1}}, \sqrt{R_{k}}\right) .
\end{aligned}
$$

4) Obtain the corrected statistics of the state by:

$$
\begin{aligned}
G_{k} & =\hat{P}_{x y}^{k \mid k-1}{\sqrt{\hat{P}_{y y}^{k \mid k-1}}}^{-T}{\sqrt{\hat{P}_{y y}^{k \mid k-1}}}^{-1}, \\
\hat{x}_{k \mid k} & =\hat{x}_{k \mid k-1}+G_{k}\left(y_{k}-\hat{y}_{k \mid k-1}\right), \\
\sqrt{\hat{P}_{x x}^{k \mid k}} & =c u\left(\left[S_{\chi}^{+}-G_{k} S_{\gamma}^{+}\right],\left[S_{\chi}^{-}-G_{k} S_{\gamma}^{-}\right], G_{k} \sqrt{R_{k}}\right) .
\end{aligned}
$$

\section{Consistent Unscented Kalman Filter variations}

In order to clarify which UKF's and SRUKF's in the literature are consistent, we put all filter variants for $U T_{1}=U T_{2}$ and $S R U T_{1}=S R U T_{2}$ in Table IV. Each filter is the resulting variant of using the proposed AdUKF (AdSRUKF) with the corresponding UT (SRUT) in the first column and with the corresponding $\sigma$-representation in the first row (e.g. the Min. Sc. AdUKF, third row and fourth column, is the result of using the AdUKF with the ScUT and the Min $\sigma$ R).

One should notice that consistent variants of the UKF (SRUKF) in the literature are particular cases of the proposed UKF (SRUKF) definitions in this work. Also, these definitions are able to provide new filter variants (e.g. the Scaled SquareRoot Unscented Kalman Filters).

\section{Computational complexity and numerical implementations}

From the computational complexity point-of-view, the UKF's most expensive operations are the square-root matrix operation of $\hat{P}_{x x}^{k-1 \mid k-1}+Q_{k}\left[\mathcal{O}\left(n^{3}\right)\right]$ and the matrix inversion of $\hat{P}_{y y}^{k \mid k-1}\left[\mathcal{O}\left(n_{y}^{3}\right)\right]$, where $n_{y}$ is the dimension of the measurement vector]. Hence, for the case in which $n_{y} \leq n$, the computational complexity of the UKF is $\mathcal{O}\left(n^{3}\right)$, which is the same as for the EKF [43]. From a numerical implementation standpoint, even though the Cholesky decomposition seems to be the most adopted method to compute the square-root matrix of the $\mathrm{CM}$ for the state, some studies indicate that other methods, such as SVD decomposition, provide better estimation quality (see [61] for more details). Some code implementations are available on-line (e.g. [62] and [63]).

For the SRUKF, the computational complexity is also $\mathcal{O}\left(n^{3}\right)$ due to the triangularization (tria \{\} ), which is its most expensive operation. One example of triangularization is the $\mathrm{QR}$ decomposition, which has different implementations: the Householder QR requires $n^{3} / 3$ floating points operations (flops) for a $n \times n$ matrix; the Givens QR requires $2 n^{3}$ flops; and the modified Gram-Schmidt QR requires $2 n^{3}$ flops [64]. However, in terms of numerical implementation, SRUKF's behave better than the non-square root forms when implemented in a machine with poor precision [42].

\section{E. Higher-order Unscented Kalman Filters}

In this work, the AdUKF and AdSRUKF were defined only with 2nd order UT's. Extensions to higher orders can be done in at least two ways. A first one is given by the following algorithm:

lth $(l>2)$ order (Gaussian) Additive UKF: Consider (1), 1) $\hat{x}_{k-1 \mid k-1}$ and $\hat{P}_{x x}^{k-1 \mid k-1}$ are given.

2) Choose $l \in \mathbb{N}, l>2$ and obtain the central moments $M_{x_{k-1 \mid k-1}}^{2}, \ldots, M_{x_{k-1 \mid k-1}}^{l}$ for $x_{k-1 \mid k-1} \sim$ $N\left(\hat{x}_{k-1 \mid k-1}, \hat{P}_{x x}^{k-1 \mid k-1}+Q_{k}\right)$.

3) Obtain the predicted statistics of the state:

$$
\begin{aligned}
& {\left[\hat{x}_{k \mid k-1}, \hat{P}_{x x}^{k \mid k-1}\right]} \\
& \quad=l U T_{1}\left(f, \hat{x}_{k-1 \mid k-1}, M_{x_{k-1 \mid k-1}}^{2}, \ldots, M_{x_{k-1 \mid k-1}}^{l}\right) .
\end{aligned}
$$

4) Obtain the central moments $M_{x_{k \mid k-1}}^{2}, \ldots, M_{x_{k \mid k-1}}^{l}$ for $x_{k \mid k-1} \sim N\left(\hat{x}_{k \mid k-1}, \hat{P}_{x x}^{k \mid k-1}+R_{k}\right)$.

5) Obtain the predicted statistics of the measurement:

$$
\begin{aligned}
& {\left[\hat{y}_{k \mid k-1}, \hat{P}_{y y}^{k \mid k-1}, \hat{P}_{x y}^{k \mid k-1}\right]=} \\
& \quad l U T_{2}\left(h, \hat{x}_{k \mid k-1}, M_{x_{k \mid k-1}}^{2}, \ldots, M_{x_{k \mid k-1}}^{l}\right) .
\end{aligned}
$$

6) Obtain the corrected statistics of the state by (7).

This approach uses the Gaussian assumption of the Kalman Filter to obtain the previous first $l$ moments of the state for each $l U T$. Generally, higher values of $l$ result in a larger number of sigma-points and better state estimation (cf. Theorem 4). Note that the higher-order UKF of [41] is a particular case of this proposed filter for the scalar case.

A second way is to propagate, at every time step, not only the mean and the covariance matrix of the state, but also its higher-order moments up to the chosen $l$ th order (a similar approach that does not use UT's is proposed by [65]). This method does not assume that the state follows a Gaussian distribution at every time step and provides a better approximation when compared to the first one, but at the cost of increased effort in developing the recursive equations and also of having a computationally more expensive algorithm.

\section{CONCLUSION}

From the study of the state-of-the-art in Unscented estimation theory, we were able to observe several important problems (Sections II-B to II-F) concerning (1) the matching order of the transformed covariance matrix (CM) (Sections II-C1 and II-E2) and the transformed cross-covariance matrix (CCM) (Sections II-C2 and II-E3) of both the Unscented 
TABLE IV

Consistent Unscented And SQuare-Root Unscented Kalman Filter VARiants For $U T_{1}=U T_{2}$ AND $S R U T_{1}=S R U T_{2}{ }^{\mathrm{a}}$

\begin{tabular}{|c|c|c|c|c|}
\hline & $\operatorname{MiSy} \sigma \mathbf{R}$ (Cor. 4) & HoMiSy $\sigma$ R (Cor. 4) & $\operatorname{Mi} \sigma \mathbf{R}$ (Th. 3) & RhoMi $\sigma \mathbf{R}$ (Cor. 5) \\
\hline UT (Def. 2) & Min. Sy. AdUKF & Hom. Min. Sy. AdUKF ${ }^{b}$ & Min. AdUKF & Rho Min. AdUKF ${ }^{g}$ \\
\hline ScUT (Def. 3) & Min. Sy. Sc. AdUKF & Hom. Min. Sy. Sc. AdUKF ${ }^{c}$ & Min. Sc. AdUKF & Rho Min. Sc. AdUKF \\
\hline SiScUT (Def. 7) & Min. Sy. Si. Sc. AdUKF & Hom. Min. Sy. Si. Sc. AdUKF ${ }^{d}$ & - & - \\
\hline SyInScUT (Def. 5) & - & Sy. Intr. Sc AdUKF ${ }^{\mathrm{e}}$ & - & - \\
\hline SRUT (Def. 6) & Min. Sy. AdSRUKF & Hom. Min. Sy. AdSRUKF ${ }^{f}$ & Min. AdSRUKF & Rho Min. AdSRUKF \\
\hline ScSRUT (Def. 7) & Min. Sy. Sc. AdSRUKF & Hom. Min. Sy. Sc. AdSRUKF & Min. Sc. AdSRUKF & Rho Min. Sc. AdSRUKF \\
\hline SiScSRUT (Def. 8) & Min. Sy. Si. Sc. AdSRUKF & Hom. Min. Sy. Si. Sc. AdSRUKF & - & - \\
\hline SyInScSRUT (Def. 9) & - & Sy. Intr. Sc. AdSRUKF ${ }^{\text {h }}$ & - & _- \\
\hline
\end{tabular}

${ }^{a}$ RhoMi $\sigma \mathrm{R}$ (Rho Minimum $\sigma$-representation) stands for the $\sigma$-representation of [12], Def. for Definition, Cor. for Corollary, Th. for Theorem, Hom. for Homogeneous, Intr. for Intrisically, Min. for Minimum, Sc. for Scaled, Si. for Simplex and Sy. for Symmetric. Each final variant of the filters without a footnote comment is a new consistent version. ${ }^{b}$ Equivalent to Tab III [1,1:3]. ${ }^{\mathrm{c}}$ Complete version of Tab III [3,4:6] with the set of Tab II [4:6]. ${ }^{\mathrm{d}}$ Complete version of Tab III [3,4:6] with the set of Tab II [1:3]. ${ }^{\mathrm{e}}$ Equivalent to Tab III $[5,1: 3] .{ }^{\mathrm{f}}$ Equivalent to the SRUKF of [4]. ${ }^{\mathrm{g}}$ Equivalent to Tab III $[2,4: 6] .{ }^{\mathrm{h}}$ Equivalent to the SRUKF of [30].

Transformation (UT) and of the Scaled Unscented Transformation (SUT); (2) multiple UKF definitions (Section II-B1); (3) issues with the reduced sets of [8], [9] and [34] (Sections II-D1, II-D2 and II-D3); (4) the conservativeness of the SUT (Section II-E1); (5) the scaling effect of the SUT on both the transformed CM and CCM (Sections II-E2 and II-E3); and (6) possibly ill-conditioned results in the square-root Unscented Kalman Filters (Section II-F1).

Focusing on discrete-time dynamical systems, we propose the solution of these problems by a systematization of the Unscented estimation theory. By doing so, we were able to (1) gather all the discrete Unscented Kalman Filters described in the literature (Section II-A); (2) formally justify the symmetric sets of sigma points (Section III-B1); (3) solve some problems within this theory (Sections III and IV); and (4) provide new Unscented Kalman Filters (Section V).

\section{ACKNOWLEDGMENT}

The authors would like to thank Mr. Pedro Henrique R. Q. A. Santana for proof-reading the paper.

\section{REFERENCES}

[1] S. J. Julier, J. K. Uhlmann, and H. F. Durrant-Whyte, "A new approach for filtering nonlinear systems," in Proc. IEEE American Control Conf. (ACC'95), 1995, pp. 1628-1632.

[2] J. Zhang and C. Xia, "State-of-charge estimation of valve regulated lead acid battery based on multi-state Unscented Kalman Filter," Int. J. Elect. Power Energy Syst., vol. 33, no. 3, pp. 472-476, Mar. 2011.

[3] C. Eberle and C. Ament, "The Unscented Kalman Filter estimates the plasma insulin from glucose measurement." BioSystems, vol. 103, no. 1, pp. 67-72, 2011.

[4] S. Holmes, G. Klein, and D. W. Murray, "An O(N(2)) Square Root Unscented Kalman Filter for Visual Simultaneous Localization and Mapping," IEEE Trans. Pattern Anal. Mach. Intell., vol. 31, no. 7, pp. 1251-63, 2009.

[5] K. Ito and K. Xiong, "Gaussian filters for nonlinear filtering problems," IEEE Trans. Autom. Control, vol. 45, no. 5, pp. 910-927, May 2000.

[6] I. Arasaratnam and S. Haykin, "Cubature Kalman Filters," IEEE Trans. Autom. Control, vol. 54, no. 6, pp. 1254-1269, 2009.

[7] S. J. Julier and J. K. Uhlmann, "Unscented filtering and nonlinear estimation," Proc. of the IEEE, vol. 92, no. 3, pp. 401-422, 2004.

[8] S. J. Julier, "Reduced sigma point filters for the propagation of means and covariances through nonlinear transformations," in Proc. IEEE American Control Conf. (ACC'02), 2002.

[9] - "The spherical simplex unscented transformation," in Proc. IEEE American Control Conf. (ACC'03), vol. 3, 2003, pp. 2430-2434.

[10] F. Gustafsson and G. Hendeby, "On nonlinear transformations of stochastic variables and its application to nonlinear filtering," in Proc. IEEE Int. Conf. Acoust. Speech Signal Process. (ICASSP'08), 2008, pp. $3617-3620$.

[11] — "Some Relations Between Extended and Unscented Kalman Filters," IEEE Trans. Signal Process., vol. 60, no. 2, pp. 545-555, 2012.
[12] H. M. Menegaz, J. Y. Ishihara, and G. A. Borges, "A new smallest sigma set for the Unscented Transform and its applications on SLAM," in Proc. IEEE Conf. Decision Control and European Control Conf. (CDC/ECC'11), 2011.

[13] Y. Wu, D. Hu, M. Wu, and X. Hu, "A Numerical-Integration Perspective on Gaussian Filters," IEEE Trans. Signal Process., vol. 54, no. 8, pp. 2910-2921, Aug. 2006.

[14] A. H. Jazwinski, Stochastic Processes and Filtering Theory. Academic Press, 1970

[15] S. Särkkä, Bayesian Filtering and Smoothing. Cambridge, UK: Cambridge University Press, 2013.

[16] R. Kalman, "A new approach to linear filtering and prediction problems," Trans. ASME J. Basic Eng., vol. 82, pp. 35-45, 1960.

[17] F. Daum, "Nonlinear filters: beyond the Kalman filter," IEEE Trans. Aerosp. Electron. Syst., vol. 20, no. 8, pp. 57-69, 2005.

[18] D. Simon, Optimal State Estimation. Kalman, $H_{\infty}$, and Nonlinear Approaches. Hoboken, New Jersey: John Wiley \& Sons, 2006.

[19] J. Duník, M. Šimandl, and O. Straka, "Unscented Kalman Filter: Aspects and Adaptive Setting of Scaling Parameter," IEEE Trans. Autom. Control, vol. 57, no. 9, pp. 2411-2416, Sep. 2012.

[20] M. Athans, R. Wishner, and A. Bertolini, "Suboptimal state estimation for continuous-time nonlinear systems from discrete noisy measurements," IEEE Trans. Autom. Control, vol. 13, no. 5, pp. 504-514, 1968.

[21] D. Alspach and H. Sorenson, "Nonlinear Bayesian estimation using Gaussian sum approximations," IEEE Trans. Autom. Control, vol. 17, no. 4, pp. 439-448, 1972

[22] M. Šimandl, J. Královec, and T. Söderström, "Advanced point-mass method for nonlinear state estimation," Automatica, vol. 42, no. 7, pp. 1133-1145, 2006.

[23] A. Doucet, N. de Freitas, and N. Gordon, Sequential Monte Carlo Methods in Pratice. Springer, 2001.

[24] O. Cappe, S. J. Godsill, and E. Moulines, "An Overview of Existing Methods and Recent Advances in Sequential Monte Carlo," Proc. of the IEEE, vol. 95, no. 5, pp. 899-924, 2007.

[25] M. F. Bugallo and P. M. Djuric, "Joint Model Selection and Parameter Estimation by Population Monte Carlo Simulation," IEEE J. Sel. Topics Signal Process., vol. 4, no. 3, pp. 526-539, 2010.

[26] D. Crisan and B. Rozovskii, The Oxford Handbook of Nonlinear Filtering. Oxford, UK: Oxford University Press, 2011.

[27] J. C. Spall, "Estimation via Markov chain Monte Carlo," IEEE Control Syst. Mag, vol. 23, no. 2, pp. 34-45, 2003.

[28] M. Nø rgaard, N. Poulsen, and O. Ravn, "New developments in state estimation for nonlinear systems," Automatica, vol. 36, no. 11, pp. 1627$1638,2000$.

[29] I. Arasaratnam, S. Haykin, and T. R. Hurd, "Cubature Kalman Filtering for Continuous-Discrete Systems: Theory and Simulations," IEEE Trans. Signal Process., vol. 58, no. 10, pp. 4977-4993, 2010.

[30] R. V. D. Merwe, "Sigma-point Kalman filters for probabilistic inference in dynamic state-space models," Ph.D. dissertation, Oregon Health \& Science University, 2004.

[31] T. Lefebvre, H. Bruyninckx, and J. D. Schutter, "Comment on âĂIJA New Method for the Nonlinear Transformation of Means and Covariances in Filters and EstimatorsâĂİ," IEEE Trans. Autom. Control, vol. 47, no. 8, pp. 1406-1408, Aug. 2002.

[32] Y. Wu, D. Hu, M. Wu, and X. Hu, "Unscented Kalman filtering for additive noise case: augmented versus nonaugmented," IEEE Signal Processing Letters, vol. 12, no. 5, pp. 357-360, May 2005. 
[33] A. Charalampidis, "Computationally Efficient Kalman Filtering For a Class of Nonlinear Systems," IEEE Trans. Autom. Control, vol. 56, no. 3, pp. 483-491, Mar. 2011.

[34] L. Wan-chun, W. Ping, and X. Xian-Ci, "A novel simplex unscented transform and filter," in Proc. IEEE Int. Symp. Comm. Inform. Technologies (ISCIT'07), 2007, pp. 926-931.

[35] E. A. Wan and R. V. D. Merwe, "The unscented Kalman filter for nonlinear estimation," in Proc. IEEE Adaptive Syst. Signal Process. Commun. Control Symp. (ASSPCCS'00), 2000, pp. 153-158.

[36] U. N. Lerner, "Hybrid Bayesian networks for reasoning about complex systems," Ph. D., Stanford University, 2002.

[37] A. C. Charalampidis and G. P. Papavassilopoulos, "Development and numerical investigation of new non-linear Kalman filter variants," IET Control Theory \& Applications, vol. 5, no. 10, p. 1155, Aug. 2011.

[38] S. J. Julier, "The scaled unscented transformation," in Proc. IEEE American Control Conf. (ACC'02), no. 2, 2002, pp. 4555-4559.

[39] - "A skewed approach to Filtering," in Proc. SPIE 3373, Conf. Signal Data Process. Small Targets, Orlando, FL, Sep. 1998, pp. $271-$ 282.

[40] S. J. Julier and J. K. Uhlmann, "Consistent debiased method for converting between polar and Cartesian coordinate systems," in Proc. SPIE, vol. 3086, 1997, pp. 110-121.

[41] D. Tenne and T. Singh, "The higher order unscented filter," in Proc. IEEE American Control Conf. (ACC'03), vol. 3, 2003, pp. 2441-2446.

[42] M. S. Grewal and A. P. Andrews, Kalman Filtering : Theory and Practice: Using Matlab. John Wiley \& Sons, 2001.

[43] R. V. D. Merwe and E. A. Wan, "The square-root unscented Kalman filter for state and parameter-estimation," in Proc. IEEE Int. Conf. Acoust. Speech Signal Process. (ICASSP'01), vol. 6, 2001, pp. 34613464 .

[44] X. Tang, X. Zhao, and X. Zhang, "The square-root spherical simplex unscented Kalman filter for state and parameter estimation," in Proc. IEEE Int. Conf. Signal Process. (ICSP'09), 2008, pp. 260-263.

[45] Z. Liu, J. Chen, X. Yao, C. Song, and Y. Wang, "An improved square root unscented Kalman filter for projectile's attitude determination," in Proc. IEEE Conf. Ind. Electron. Applicat. (ICIEA), 2010, pp. 1747 1751.

[46] S. Särkkä, "On Unscented Kalman Filtering for State Estimation of Continuous-Time Nonlinear Systems," IEEE Trans. Autom. Control, vol. 52, no. 9, pp. 1631-1641, 2007.

[47] A. Björck, Numerical methods for least squares problems. SIAM, 1996.

[48] I. Arasaratnam and S. Haykin, "Square-Root Quadrature Kalman Filtering," IEEE Trans. Signal Process., vol. 56, no. 6, pp. 2589-2593, 2008.

[49] M. L. Carneiro, P. H. P. de Carvalho, N. Deltimple, L. d. C. Brito, L. R. de Menezes, E. Kerherve, S. G. de Araujo, and A. S. Rocha, "Doherty amplifier optimization using robust genetic algorithm and Unscented Transform," in Proc. IEEE Int. New Circuits Syst. Conf. (NEWCAS), 2011, pp. 77-80.

[50] S. Särkkä, "Continuous-time and continuous-discrete-time unscented Rauch-Tung-Striebel smoothers," Signal Process., vol. 90, no. 1, pp. 225-235, 2010.

[51] V. P. Nia, "Gauss-Hermite Quadrature: Numerical or Statistical Method?" in Proc. Iranian Stat. Conf., 2006, pp. 209-215.

[52] T. J. Giese, "Numerical quadrature," Rutgers, The State University of New Jersey, Tech. Rep., 2006.

[53] P. Jäckel, "A note on multivariate Gauss-Hermite quadrature," Oxford University, Tech. Rep. May, 2005.

[54] A. I. Hascelik, "Gauss quadrature rules for a generalized Hermite weight function," Applied Math. Comput., vol. 180, no. 1, pp. 86-96, 2006.

[55] I. Arasaratnam, S. Haykin, and R. J. Elliott, "Discrete-Time Nonlinear Filtering Algorithms Using Gauss-Hermite Quadrature," Proc. of the IEEE, vol. 95, no. 5, pp. 953-977, 2007.

[56] P. J. Davis and P. Rabinowitz, Methods of Numerical Integration, 2nd ed., ser. Computer Science and Applied Mathematics. San Diego, CA: Academic Press, 1984.

[57] D. P. Kroese, T. Taimre, and Z. I. Botev, Handbook of Monte Carlo Methods. John Wiley \& Sons, 2011.

[58] A. C. Charalampidis and G. P. Papavassilopoulos, "Kalman Filtering for a Generalized Class of Nonlinear Systems and a New Gaussian Quadrature Technique," IEEE Trans. Autom. Control, vol. 57, no. 11, pp. 2967-2973, Nov. 2012.

[59] S. J. Julier, J. K. Uhlmann, and H. F. Durrant-Whyte, "A New Method for the Nonlinear Transformation of Means and Covariances in Filters and Estimators," IEEE Trans. Autom. Control, vol. 45, no. 3, pp. 477482,2000
[60] A. Sakai and Y. Kuroda, "Discriminatively Trained Unscented Kalman Filter for Mobile Robot Localization," J. Advanced Research Mech. Eng., vol. 1, no. 3, pp. 153-161, 2010.

[61] O. Straka, J. Duník, M. Simandl, and J. HavlIk, "Aspects and Comparison of Matrix Decompositions in Unscented Kalman Filter," in Proc. IEEE American Control Conf. (ACC'13), Washington, DC, Jun. 2013, pp. 3075-3080.

[62] Y. Cao, "ukf.m." [Online]. Available: http://www.mathworks.com/ matlabcentral/fileexchange/18217-learning-the-unscented-kalman-filter.

[63] D. Dyer, "ukf.cpp." [Online]. Available: https://github.com/sfwa/ukf/ blob/master/src/ukf.cpp.

[64] G. H. Golub and C. F. V. Loan, Matrix Computations, 4th ed. Baltimore, Maryland: John Hopkins University Press, 2013.

[65] A. Benavoli, "The Generalized Moment-Based Filter," IEEE Trans. Autom. Control, vol. 58, no. 10, pp. 2642-2647, Oct. 2013.

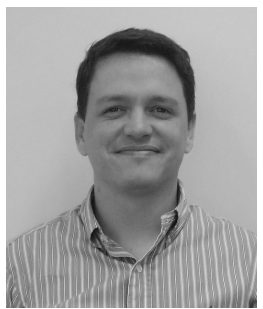

Henrique M. T. Mennegaz received the B.S. degree in electrical engineering and the M.S. degree in engineering of electronic systems and automation from the University of Brasília (UnB), Brazil, in 2007 and 2011, respectively, where he is currently pursuing the Ph.D. degree.

His major field of study is filtering of nonlinear dynamic systems and their applications.

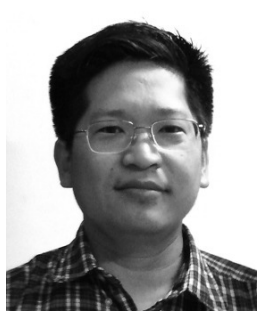

João Y. Ishihara received the Ph.D. degree in Electrical Engineering from the University of São Paulo, Brazil, in 1998

$\mathrm{He}$ is currently an Associate Professor at the University of Brasília, Brazil. His research interests include robust filtering and control theory, singular systems, and robotics.

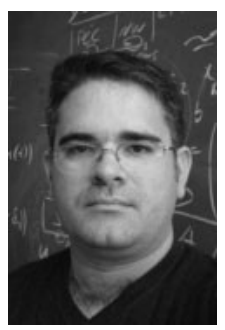

Geovany A. Borges received the Ph.D. degree from the Université Montpellier 2, France, in 2002.

$\mathrm{He}$ is currently an Associate Professor at the Universidade de Brasília, Brazil. His main research interests are robotics, stochastic filtering, and estimation

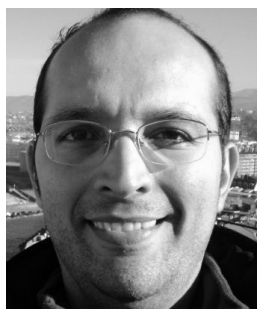

Alessandro N. Vargas received $\mathrm{Ph} . \mathrm{D}$. degree in electrical engineering from the University of Campinas, Brazil, in 2009.

$\mathrm{He}$ is currently an Associate Professor at the Universidade Tecnológica Federal do Paraná, Brazil. His research interests include stochastic systems control and their applications. 